\title{
On square functions with independent increments and Sobolev spaces on the line
}

\author{
Julià Cufí $^{1} \cdot$ Artur Nicolau $^{1} \cdot$ Andreas Seeger $^{2}$. \\ Joan Verdera ${ }^{1}$
}

Received: 20 February 2017 / Accepted: 16 October 2017 / Published online: 7 November 2017

(C) Fondazione Annali di Matematica Pura ed Applicata and Springer-Verlag GmbH Germany 2017

\begin{abstract}
We prove a characterization of some $L^{p}$-Sobolev spaces involving the quadratic symmetrization of the Calderón commutator kernel, which is related to a square function with differences of difference quotients. An endpoint weak type estimate is established for functions in homogeneous Hardy-Sobolev spaces $\dot{H}_{\alpha}^{1}$. We also use a local version of this square function to characterize pointwise differentiability for functions in the Zygmund class.
\end{abstract}

Keywords Hardy space $\cdot$ Sobolev space $\cdot$ Square function · Zygmund class

Mathematics Subject Classification 42B15 - 42B30 - 42A99

\section{Introduction}

In this paper we give a characterization of Sobolev spaces on the real line by a square function which appears in some proofs of the $L^{2}$-boundedness of the first Calderón commutator [23] and the Cauchy integral on a Lipschitz or chord arc curve [11,23]. Moreover, a local version of this square function can be used to describe the set of points where a given function is pointwise differentiable.

Joan Verdera

jvm@mat.uab.cat

Julià Cufí

jcufi@mat.uab.cat

Artur Nicolau

artur@mat.uab.cat

Andreas Seeger

seeger@math.wisc.edu

1 Departament de Matemàtiques, Universitat Autònoma de Barcelona, 08193 Bellaterra (Barcelona), Catalonia

2 Department of Mathematics, University of Wisconsin, 480 Lincoln Drive, Madison, WI 53706, USA 
Our square function acts on functions on the real line and involves the difference of two difference quotients with increments $s$ and $t$. Define

$$
S_{\alpha} f(x)=\left(\iint_{\mathbb{R} \times \mathbb{R}}\left|\frac{f(x+s)-f(x)}{s}-\frac{f(x+t)-f(x)}{t}\right|^{2} \frac{\mathrm{d} s \mathrm{~d} t}{|s-t|^{2 \alpha}}\right)^{1 / 2} .
$$

This square function is a rough relative of the more standard Marcinkiewicz square function associated with second differences,

$$
G_{\alpha} f(x)=\left(\int_{0}^{\infty} \frac{|f(x+2 t)-2 f(x+t)+f(x)|^{2}}{t^{1+2 \alpha}} \mathrm{d} t\right)^{1 / 2},
$$

which was introduced for $\alpha=1$ by Marcinkiewicz to investigate questions about pointwise differentiability (see [18]). In Sect. 3 we prove that for $\alpha \geq 0$ and $f \in L^{2}(\mathbb{R})$ there is a pointwise majorization

$$
G_{\alpha} f(x) \leq C(\alpha) S_{\alpha} f(x) .
$$

We shall prove sharp results on mapping properties of $S_{\alpha}$ when acting in $L^{p}$-Sobolev spaces. Our starting point is the identity $\left\|S_{1} f\right\|_{2}=c\left\|f^{\prime}\right\|_{2}$, proved in [11] by an application of Plancherel's theorem. We aim for an analogous characterizations of other homogeneous Sobolev spaces $\dot{H}_{\alpha}^{p}$, with $p \neq 2$ and suitable $\alpha$. It is proved in Sect. 4 that such a characterization is limited to the range $1 / 2<\alpha<3 / 2$. Recall that, for $1<p<\infty$, the (semi)-norm on $\dot{H}_{\alpha}^{p}$ is given by $\left\|\mathcal{D}^{\alpha} f\right\|_{p}$, where $\mathcal{D}^{\alpha}$ denotes the Riesz derivative operator of order $\alpha$; it is defined by $\widehat{\mathcal{D}^{\alpha} g}(\xi)=|\xi|^{\alpha} \widehat{g}(\xi)$, at least for Schwartz functions whose Fourier transform is compactly supported in $\mathbb{R} \backslash\{0\}$. Of course $\mathcal{D}^{\alpha}$ is the inverse of the Riesz potential operator $I^{\alpha}=\mathcal{D}^{-\alpha}$, given for $0<\alpha<1$ by $I^{\alpha} f=\gamma(\alpha) \cdot\left(f *|x|^{\alpha-1}\right)$, with $\gamma(\alpha)$ a constant, and defined for other $\alpha$ by analytic continuation. The space $\dot{H}_{\alpha}^{p}$ consists of all distributions which are Riesz potentials of order $\alpha$ of $L^{p}$ functions. See [16,17,21,22] for more on these spaces.

Theorem 1.1 Let $1<p<\infty, 1 / 2<\alpha<3 / 2$. Then,

$$
\left\|\mathcal{D}^{\alpha} f\right\|_{L^{p}(\mathbb{R})} \approx\left\|S_{\alpha} f\right\|_{L^{p}(\mathbb{R})} .
$$

Here the implicit constants depend only on $p$ and $\alpha$.

In contrast we have the larger range $\max \{1 / p-1 / 2,0\}<\alpha<2$ in the known equivalence $\left\|\mathcal{D}^{\alpha} f\right\|_{p} \approx\left\|G_{\alpha} f\right\|_{p}$ for the Marcinkiewicz square function, see [20].

The proof of Theorem 1.1 is immediately reduced to the equivalence

$$
\|f\|_{L^{p}(\mathbb{R})} \approx\left\|S_{\alpha}\left(\mathcal{D}^{-\alpha} f\right)\right\|_{L^{p}(\mathbb{R})} .
$$

It is natural to ask whether this result extends to $p=1$ in the sense of a characterization for the homogeneous Hardy-Sobolev spaces $\dot{H}_{\alpha}^{1}$. The vector-valued operator associated with $f \mapsto S_{\alpha}\left(\mathcal{D}^{-\alpha} f\right)$ is not covered by the standard theory in [1] but should be considered as a rough singular integral in the spirit of [5].

We let $H^{1}$ stand for the usual Hardy space on the line, that is, for the set of functions $f$ in $L^{1}$ such that the Hilbert transform of $f$ is also in $L^{1}$. It turns out that the strong $H^{1} \rightarrow L^{1}$ boundedness of $f \rightarrow S_{\alpha}\left(\mathcal{D}^{-\alpha}\right) f$ fails; this is in contrast to a positive result for the Marcinkiewicz square function, namely $\left\|G_{\alpha}\left(\mathcal{D}^{-\alpha} f\right)\right\|_{1} \lesssim\|f\|_{H^{1}}$ for $1 / 2<\alpha<2$. See, e.g., [21, §3.5.3]. The following $H^{1} \rightarrow L^{1, \infty}$ endpoint result for $f \mapsto S^{\alpha}\left(\mathcal{D}^{-\alpha} f\right)$ is optimal in the sense that $L^{1, \infty}$ cannot be replaced by a Lorentz space $L^{1, q}$ with $q<\infty$, see Sect. 4.3. 
Theorem 1.2 Let $1 / 2<\alpha<3 / 2$. Then, for all $f$ in the homogeneous Hardy-Sobolev space $\dot{H}_{\alpha}^{1}$ and all $\lambda>0$,

$$
\operatorname{meas}\left(\left\{x: S_{\alpha} f(x)>\lambda\right\}\right) \leq C_{\alpha} \lambda^{-1}\left\|\mathcal{D}^{\alpha} f\right\|_{H^{1}} .
$$

The statement above for $\alpha=1$ can be restated in terms of the first derivative, using the Hilbert transform.

Corollary 1.3 (i) For $f \in L^{p}, 1<p<\infty,\left\|S_{1} f\right\|_{p} \approx\left\|f^{\prime}\right\|_{p}$.

(ii) If $f^{\prime} \in H^{1}$ then meas $\left(\left\{x: S_{1} f(x)>\lambda\right\}\right) \leq C \lambda^{-1}\left\|f^{\prime}\right\|_{H^{1}}$.

In Sect. 4.4 we show that the condition $f^{\prime} \in H^{1}$ in the second part of Corollary 1.3 cannot be replaced by $f^{\prime} \in L^{1}$, and formulate a related open question for the Riesz derivatives.

We shall also consider a local version of the square function $S_{1}$ in order to study pointwise differentiability. Recall that a bounded function $f: \mathbb{R} \rightarrow \mathbb{R}$ is in the Zygmund class $\Lambda_{*}$ (also known as the homogeneous Besov space $\dot{B}_{1}^{\infty, \infty}$ ) if there exists a constant $c=c(f)>0$ such that

$$
|f(x+h)+f(x-h)-2 f(x)| \leq c|h|, \quad x, h \in \mathbb{R} .
$$

The infimum of the constants $c$ satisfying the above inequality is denoted by $\|f\|_{\Lambda_{*}}$. Functions in the Zygmund class may be nowhere differentiable. For example, the Weierstrass function $f(x)=\sum_{n=1}^{\infty} b^{-n} \cos \left(b^{n} x\right)$, where $b>1$, is nowhere differentiable and belongs to $\Lambda_{*}$. It turns out that a local version of $S_{1}$ characterizes the almost everywhere differentiability of functions in the Zygmund class, very much in the spirit of a classical theorem of Stein and Zygmund [19] which uses a local version of the Marcinkiewicz square function $G_{1}$. Our result reads as follows.

Theorem 1.4 Let $f: \mathbb{R} \rightarrow \mathbb{R}$ belong to the Zygmund class $\Lambda_{*}$. Then, the set of points $x \in \mathbb{R}$ such that

$$
\iint_{|t|+|s|<1}\left|\frac{f(x+s)-f(x)}{s}-\frac{f(x+t)-f(x)}{t}\right|^{2} \frac{\mathrm{d} s \mathrm{~d} t}{|s-t|^{2}}<\infty
$$

coincides, except possibly for a set of Lebesgue measure zero, with the set of points where $f$ is differentiable.

In view of the pointwise inequality $G_{1} f \lesssim S_{1} f$ the main point of Theorem 1.4 is that almost everywhere the pointwise differentiability for functions in the Zygmund class implies the finiteness of the rough square function. For general functions in $L_{\mathrm{loc}}^{2}$ this implication fails, see Sect. 9.1.2. Theorem 1.4 will be obtained as a simple consequence of a more general result formulated as Theorem 9.3.

This paper In Sect. 2 we discuss the connection with quadratic symmetrizations of Calderón commutators and with the Cauchy integral. In Sect. 3 we prove a generalization of the pointwise majorization result (3). In Sect. 4 we briefly discuss necessary conditions for Theorems 1.1 and 1.2. In Sect. 5 we prove the lower bound in Theorem 1.1, namely $\left\|\mathcal{D}^{\alpha} f\right\|_{p} \lesssim\left\|S_{\alpha} f\right\|_{p}, 1<p<\infty$. In Sect. 6 we discuss basic decompositions of our operators and prove some refined $L^{2}$ bounds that are crucial for the proofs of Theorems 1.1 and 1.2. In Sect. 7 we prove the endpoint Theorem 1.2. In Sect. 8 we quickly discuss various approaches to Theorem 1.1 via interpolation arguments. In Sect. 9 we state and prove the results on pointwise differentiability. 


\section{Relation with Calderón commutators}

For suitable functions $A: \mathbb{R} \rightarrow \mathbb{C}$ consider the first Calderón commutator $\mathcal{C}_{A}$ whose Schwartz kernel $\mathcal{K}_{A}$ is given by

$$
\mathcal{K}_{A}(x, y)=p \cdot v \cdot \frac{A(y)-A(x)}{(x-y)^{2}} .
$$

Calderón [3] proved the $L^{2}(\mathbb{R})$ boundedness of $\mathcal{C}_{A}$ for Lipschitz functions $A$; subsequently many other proofs were discovered. Here we are motivated by the proof in [23] which uses a symmetrization technique based on the three term quadratic symmetrization

$$
\begin{aligned}
& \operatorname{Sym}\left[\mathcal{K}_{A}\right](x, y, z) \\
& \left.\quad:=\mathcal{K}_{A}(x, y) \mathcal{K}_{A}(x, z)\right)+\mathcal{K}_{A}(y, z) \mathcal{K}_{A}(y, x)+\mathcal{K}_{A}(z, x) \mathcal{K}_{A}(z, y),
\end{aligned}
$$

which is well defined as a function on

$$
G=\{(x, y, z) \in \mathbb{R} \times \mathbb{R} \times \mathbb{R}: x \neq y, x \neq z, y \neq z\} .
$$

We have the following elementary but crucial identity ([23]).

Lemma 2.1 For $(x, y, z) \in G$,

$$
\operatorname{Sym}\left[\mathcal{K}_{A}\right](x, y, z)=\frac{1}{(z-y)^{2}}\left(\frac{A(y)-A(x)}{y-x}-\frac{A(z)-A(x)}{z-x}\right)^{2} .
$$

Proof We use the notation $D_{a b}:=A(a)-A(b)$. For all $(x, y, z)$ we compute

$$
\begin{aligned}
(x-y)^{2}(x-z)^{2}(y-z)^{2} \operatorname{Sym}\left[\mathcal{K}_{A}\right](x, y, z) \\
=(y-z)^{2} D_{x y} D_{x z}+(z-x)^{2} D_{y z} D_{y x}+(x-y)^{2} D_{z x} D_{z y} \\
=x^{2} D_{y z}\left(D_{y x}-D_{z x}\right)+y^{2} D_{z x}\left(D_{z y}-D_{x y}\right)+z^{2} D_{x y}\left(D_{x z}-D_{y z}\right) \\
\quad-2 y z D_{x y} D_{x z}-2 z x D_{y z} D_{y x}-2 x y D_{z x} D_{z y}
\end{aligned}
$$

and using $D_{a c}-D_{b c}=D_{a b}$ we see that $(x-y)^{2}(x-z)^{2}(y-z)^{2} \operatorname{Sym}\left[\mathcal{K}_{A}\right](x, y, z)$ is equal to

$$
x^{2} D_{y z}^{2}+y^{2} D_{z x}^{2}+z^{2} D_{x y}^{2}-2 x y D_{x z} D_{y z}-2 x z D_{x y} D_{z y}-2 y z D_{y x} D_{z x} .
$$

Now it turns out that this expression is also equal to

$$
\left.\left((z-x) D_{y x}-(y-x) D_{z x}\right)\right)^{2} .
$$

Indeed the last display equals

$$
\begin{aligned}
& (z-x)^{2} D_{y x}^{2}+(y-x)^{2} D_{z x}^{2}-2(z-x)(y-x) D_{y x} D_{z x} \\
& =z^{2} D_{y z}^{2}+y^{2} D_{z x}^{2}-2 z y D_{y x} D_{z x}+R
\end{aligned}
$$

where $R=-2 x z\left(D_{y x}^{2}-D_{y x} D_{z x}\right)-2 x y\left(D_{z x}^{2}-D_{y x} D_{z x}\right)-x^{2}\left(D_{y x}-D_{z x}\right)^{2}$. Now use $D_{y x}-D_{z x}=D_{y z}$ and conclude that (6a) and (6b) coincide. This yields the asserted formula.

Using Lemma 2.1 the result of Theorem 1.1 can now be written in terms of the quadratic symmetrization: 
Corollary 2.2 For $1<p<\infty, 1 / 2<\alpha<3 / 2$,

$$
\left\|\mathcal{D}^{\alpha} A\right\|_{p}^{p} \approx \int\left(\iint \frac{\left|\operatorname{Sym}\left[\mathcal{K}_{A}\right](x, y, z)\right|}{|y-z|^{2 \alpha-2}} \mathrm{~d} y \mathrm{~d} z\right)^{p / 2} \mathrm{~d} x .
$$

As mentioned before, for $\alpha=1, p=2$ the equivalence of norms becomes an identity (noted in [11]). Indeed (5) and a Fourier transform calculation using Plancherel's theorem yield

$$
\iiint_{\mathbb{R} \times \mathbb{R} \times \mathbb{R}}\left|\operatorname{Sym}\left[\mathcal{K}_{A}\right](x, y, z)\right| \mathrm{d} x \mathrm{~d} y \mathrm{~d} z=c \int_{\mathbb{R}}\left|A^{\prime}(x)\right|^{2} \mathrm{~d} x .
$$

This argument can also be applied to the cases $1 / 2<\alpha<3 / 2$. In [23] it is explained how (7) can be used to prove the $L^{2}$ boundedness of $\mathcal{C}_{A}$ when $A$ is Lipschitz: one checks the assumptions of the $T(1)$ theorem of David and Journé. In fact, the $T(1)$ can be bypassed by a simple argument, which reduces matters to the $H^{1}-B M O$ duality.

Moreover, in [23] it is shown that the action of the Cauchy integral operator for the Lipschitz graph on characteristic functions of intervals is majorized by the action of the first Calderón commutator. The argument uses crucially the concept of Menger curvature which is controlled by $\operatorname{Sym}\left[K_{A}\right]$. To be specific, the Menger curvature function associated to the graph $\mathfrak{z}(x)=(x, A(x))$ is defined by $(R(x, y, z))^{-1}$ where $R(x, y, z)$ is the radius of the circle through the points $\mathfrak{z}(x), \mathfrak{z}(y), \mathfrak{z}(z)$ (the Menger curvature is zero if the three points lie on a straight line). The crucial identity is

$$
\frac{1}{R(x, y, z)}=\frac{4 \operatorname{area}(\mathcal{T}(x, y, z))}{|\mathfrak{z}(y)-\mathfrak{z}(x)||\mathfrak{z}(z)-\mathfrak{z}(y)||\mathfrak{z}(x)-\mathfrak{z}(z)|}
$$

where $\mathcal{T}(x, y, z)$ is the triangle with vertices $\mathfrak{z}(x), \mathfrak{z}(y), \mathfrak{z}(z)$. The identity implies, after using $|\mathfrak{z}(a)-\mathfrak{z}(b)| \geq|a-b|$ and (5), the inequality

$$
\frac{1}{R(x, y, z)} \leq 2\left|\operatorname{Sym}\left[\mathcal{K}_{A}\right](x, y, z)\right|^{1 / 2} \text {. }
$$

See [11,23] for more on the proof of the $L^{2}$ boundedness of the Cauchy integral operator based on Menger curvature. We do not emphasize Menger curvature in this paper since, while the inequality (8) is efficient when $A$ is a Lipschitz function (as then $|\mathfrak{z}(a)-\mathfrak{z}(b) \approx| a-b \mid$ ), it may be wasteful for the Sobolev classes of functions we are interested here.

\section{Comparison with Marcinkiewicz type square functions}

Given $f \in L^{2}(\mathbb{R})$ and $m \in \mathbb{R}$, consider the following square functions defined for $x \in \mathbb{R}$ by

$$
\mathcal{G}_{\alpha, m} f(x)=\left(\int_{\mathbb{R}}\left|\frac{f(x+m t)-f(x)}{m t}-\frac{f(x+t)-f(x)}{t}\right|^{2} \frac{\mathrm{d} t}{|t|^{2 \alpha-1}}\right)^{1 / 2} .
$$

The square function $S_{\alpha}$ can be recovered from the $\mathcal{G}_{\alpha, m}$ using the identity

$$
S_{\alpha} f(x)^{2}=2 \int_{|m|>1} \mathcal{G}_{\alpha, m} f(x)^{2} \frac{\mathrm{d} m}{|m-1|^{2 \alpha}}
$$

which follows by the change of variables $s=m t$ for $|s| \geq|t|$, and symmetry considerations. Conversely, the next lemma shows a pointwise domination of $\mathcal{G}_{\alpha, m} f$ in terms of $S_{\alpha} f$, for every $m>1$. For $m=2$ we have

$$
\mathcal{G}_{\alpha, 2} f(x)=\frac{G_{\alpha} f(x)}{2}
$$


for the Marcinkiewicz square function $G_{\alpha} f$ and thus recover the pointwise inequality (3) stated in the introduction.

Lemma 3.1 Let $\alpha \geq 0$. Then, for $m>1$, there exists a constant $C_{\alpha, m}$ such that for all $f \in L^{2}(\mathbb{R})$

$$
\mathcal{G}_{\alpha, m} f(x) \leq C_{\alpha, m} S_{\alpha} f(x), x \in \mathbb{R} .
$$

Proof Fix $s \in \mathbb{R}$ and $m>1$. We have

$$
\begin{aligned}
\mathcal{G}_{\alpha, m} f(x)^{2} \leq & 2 \int_{\mathbb{R}}\left|\frac{f(x+m t)-f(x)}{m t}-\frac{f(x+m t / s)-f(x)}{m t / s}\right|^{2} \frac{\mathrm{d} t}{|t|^{2 \alpha-1}} \\
& +2 \int_{\mathbb{R}}\left|\frac{f(x+m t / s)-f(x)}{m t / s}-\frac{f(x+t)-f(x)}{t}\right|^{2} \frac{\mathrm{d} t}{|t|^{2 \alpha-1}} .
\end{aligned}
$$

The change of variable $m t / s=u$ shows that the first term is equal to $2(s / m)^{2-2 \alpha} \mathcal{G}_{\alpha, s} f(x)^{2}$. Hence,

$$
\mathcal{G}_{\alpha, m} f(x)^{2} \leq 2(s / m)^{2-2 \alpha} \mathcal{G}_{\alpha, s} f(x)^{2}+2 \mathcal{G}_{\alpha, m / s} f(x)^{2} .
$$

Observe that the interval $[1, m]$ is invariant under the change of variable $s \mapsto m / s$. Integrating with respect to the measure $\mathrm{d} s / s$ yields

$$
\begin{aligned}
\mathcal{G}_{\alpha, m} f(x)^{2} \log m & =\int_{1}^{m} \mathcal{G}_{\alpha, m} f(x)^{2} \frac{\mathrm{d} s}{s} \\
& \leq 2 \int_{1}^{m}\left[(s / m)^{2-2 \alpha} \mathcal{G}_{\alpha, s} f(x)^{2}+\mathcal{G}_{\alpha, m / s} f(x)^{2}\right] \frac{\mathrm{d} s}{s} \\
& =2 \int_{1}^{m} \mathcal{G}_{\alpha, s} f(x)^{2}\left(\frac{s^{2-2 \alpha}}{m^{2-2 \alpha}}+1\right) \frac{\mathrm{d} s}{s} \\
& \leq 2 A_{\alpha, m} \int_{1}^{m} \mathcal{G}_{\alpha, s} f(x)^{2} \frac{\mathrm{d} s}{(s-1)^{2 \alpha}}
\end{aligned}
$$

where $A_{\alpha, m}:=\sup _{1 \leq s \leq m}\left((s / m)^{2-2 \alpha}+1\right) s^{-1}(s-1)^{2 \alpha}$ is clearly finite for $\alpha \geq 0$. Now by the identity (10) we obtain

$$
\mathcal{G}_{\alpha, m} f(x) \leq\left(\frac{A_{\alpha, m}}{\log m}\right)^{1 / 2} S_{\alpha} f(x) .
$$

\section{Necessary conditions}

We show that our characterization fails to extend to the Hardy-Sobolev spaces (corresponding to $p=1$ ) and that the condition $1 / 2<\alpha<3 / 2$ in Theorem 1.1 is necessary. In what follows we use the notation

$$
\Delta_{s} f(x)=f(x+s)-f(x)
$$

for the difference operator with increment $s$.

The restriction $1 / 2<\alpha<3 / 2$ is known to be necessary in other similar contexts. For instance, that $\alpha<3 / 2$ is a consequence of Proposition 2 in [2] applied to $s^{-1} \Delta_{s} f(x)$. However we present below a direct argument for the case at hand. 


\subsection{The condition $\alpha>1 / 2$}

Suppose that $0<\alpha \leq 1 / 2$. Consider $f \in C_{c}^{\infty}$ with vanishing moments up to order 2 , with the property that $f(x)=1$ for $x \in[0,1]$ and $f$ is supported in $(-3 / 2,3 / 2)$. Then, $f \in \dot{H}_{\alpha}^{p}$ for $p \geq 1$. Notice that

$$
s^{-1} \Delta_{s} f(x)-t^{-1} \Delta_{t} f(x)=-s^{-1}+t^{-1} \text { for } x \in[0,1], t \in\left[\frac{3}{2}, 2\right], s>4,
$$

and thus, for $x \in[0,1]$,

$$
S_{\alpha} f(x) \geq \frac{1}{4}\left(\int_{s=4}^{\infty}|s-2|^{-2 \alpha} \mathrm{d} s\right)^{1 / 2}=\infty, \text { if } \alpha \leq 1 / 2 .
$$

Thus we need to have $\alpha>1 / 2$.

\subsection{The condition $\alpha<3 / 2$}

Let $f \in C_{c}^{\infty}$ with vanishing moments up to order 2 and satisfying $f(x)=x^{2}$ for $|x| \leq 4$. As above, $f \in \dot{H}_{\alpha}^{p}$ for $p \geq 1$. Now

$$
s^{-1} \Delta_{s} f(x)-t^{-1} \Delta_{t} f(x)=\int_{0}^{1} f^{\prime}(x+u s)-f^{\prime}(x+u t) \mathrm{d} u
$$

so that $s^{-1} \Delta_{s} f(x)-t^{-1} \Delta_{t} f(x)=s-t$ if $|x| \leq 1,|s+t| \leq 1,|s-t| \leq 1$, and we get

$$
S_{\alpha} f(x) \geq\left(\iint_{\substack{|s-t| \leq 1 \\|s+t| \leq 1}}|s-t|^{2-2 \alpha} \mathrm{d} s \mathrm{~d} t\right)^{1 / 2} \quad \text { for }|x| \leq 1 .
$$

Hence, if $\alpha \geq 3 / 2$ then $S_{\alpha} f(x)=\infty$ for $|x| \leq 1$ which shows the necessity of the condition $\alpha<3 / 2$.

\subsection{Failure of the strong type Hardy space bound}

We show that for functions in the homogeneous Hardy-Sobolev spaces $\dot{H}_{\alpha}^{1}$ the square function $S_{\alpha} f$ may fail to be in $L^{1}$, or even in any Lorentz space $L^{1, q}$ with $q<\infty$.

Let $f$ be an odd smooth function with compact support in $(-2,2)$ such that $f(y)=1$ for $y \in[1 / 2,1]$. Using dyadic frequency decompositions one can show that $\mathcal{D}^{\alpha} f \in H^{1}(\mathbb{R})$ for $\alpha \geq 0$. Let $x>2$. We then have

$$
\begin{aligned}
& \Delta_{s} f(x)=1 \quad \text { if }-x+1 / 2 \leq s \leq-x+1, \\
& \Delta_{t} f(x)=-1 \quad \text { if }-x-1 \leq t \leq-x-1 / 2 .
\end{aligned}
$$

Hence, by (1) we get for $x>2$,

$$
S_{\alpha} f(x) \geq\left(\int_{s=-x+1 / 2}^{-x+1} \int_{t=-x-1}^{-x-1 / 2} \frac{\left|s^{-1}+t^{-1}\right|^{2}}{|s-t|^{2 \alpha}} \mathrm{d} v \mathrm{~d} w\right)^{1 / 2} \geq \frac{1}{2(x-1)}
$$

and thus $S_{\alpha} f \notin L^{1, q}(\mathbb{R})$ for $q<\infty$.

\subsection{Failure of a weak type $(1,1)$ bound}

We prove the statement given after Corollary 1.3 and show that there is a sequence of functions $f_{j}$ such that $\left\|f_{j}^{\prime}\right\|_{1}=O(1)$ and the $S_{1} f_{j}$ are unbounded in $L^{1, \infty}$. 
Define $f_{j}(x)=0$ for $x \leq 0, f_{j}(x)=j x$, for $0 \leq x \leq j^{-1}$ and $f_{j}(x)=1$ for $x>j^{-1}$ so that $f_{j}$ is a regularized version of the Heaviside function. We have $f_{j}^{\prime}=j \mathbb{1}_{\left[0, j^{-1}\right]}$ so that $\left\|f_{j}^{\prime}\right\|_{1}=1$.

Let now $-3 / 4 \leq x \leq-1 / 2$. Then, $f_{j}(x)=0$ and $f_{j}(x+s)=1$ if $-x+1 / j \leq s \leq 1$, moreover $f_{j}(x+t)=0$ for $j^{-1} \leq t \leq-x$. We thus get, for $j \geq 100$,

$$
\begin{aligned}
S_{1} f(x) & \geq\left(\int_{s=-x+j^{-1}}^{1} s^{-2} \int_{1 / j}^{-x}(s-t)^{-2} \mathrm{~d} t \mathrm{~d} s\right)^{1 / 2} \\
& \geq c\left(\int_{-x+j^{-1}}^{1}\left((s+x)^{-1}-\left(s-j^{-1}\right)^{-1}\right) \mathrm{d} s\right)^{1 / 2} \geq c^{\prime}(\log j-C)^{1 / 2} .
\end{aligned}
$$

Hence, for large $j$ and small $c$

$$
\operatorname{meas}\left\{x: S_{1} f_{j}(x) \geq c \sqrt{\log j}\right\} \geq 1 / 4
$$

which shows $\left\|S_{1} f_{j}\right\|_{L^{1, \infty}} /\left\|f_{j}^{\prime}\right\|_{1} \gtrsim \sqrt{\log j}$.

Open problem: It would be interesting to explore what happens if the ordinary derivative $f^{\prime}$ is replaced by the Riesz derivative $\mathcal{D}^{1} f$. More generally, does the weak type $(1,1)$ inequality $\left\|S_{\alpha} f\right\|_{L^{1, \infty}} \lesssim\left\|\mathcal{D}^{\alpha} f\right\|_{L^{1}}$ hold for $1 / 2<\alpha<3 / 2$ ?

\section{$5 L^{p}$ converse estimates}

It is our objective to prove the converse estimate

$$
\|f\|_{p} \leq C_{\alpha, p}\left\|S_{\alpha}\left(\mathcal{D}^{-\alpha} f\right)\right\|_{p}
$$

for $1<p<\infty$. There is no restriction on $\alpha$ in this part of the proof.

First consider the function $\rho(s)=s^{-1}\left(e^{i s}-1\right)$ and observe that

$$
\rho(s)-\rho(t)=\frac{1}{2}(t-s)+E(s, t)
$$

where $|E(s, t)| \leq C(|s|+|t|)|s-t|$ for $|s|,|t| \leq 1$. Let

$$
R_{\varepsilon}=\{(s, t): \varepsilon<s<2 \varepsilon, \varepsilon / 10<|s-t|<\varepsilon / 5\} ;
$$

then for sufficiently small $\varepsilon>0$ the function

$$
m(\xi)=\frac{1}{\left|R_{\varepsilon}\right|} \iint_{R_{\varepsilon}}\left(\frac{e^{i s \xi}-1}{s}-\frac{e^{i t \xi}-1}{t}\right)|s-t|^{-\alpha} \mathrm{d} s \mathrm{~d} t
$$

is smooth on $[-4,-1 / 4] \cup[1 / 4,4]$, and moreover

$$
|m(\xi)| \geq C_{\varepsilon, \alpha}>0, \quad 1 / 4 \leq|\xi| \leq 4 .
$$

Let $\varphi$ be supported in $(1 / 2,2)$ such that $\sum_{k \in \mathbb{Z}} \varphi\left(2^{-k}(|\xi|)\right)=1$ for all $\xi \neq 0$. Let $\widetilde{\varphi} \in C^{\infty}$ be supported in $(1 / 4,4)$ such that $\widetilde{\varphi}=1$ on $[1 / 2,2]$. Then, $\xi \mapsto \widetilde{\varphi}(|\xi|) / m(\xi)$ is a $C_{c}^{\infty}$ function with support in $\{\xi: 1 / 4<|\xi|<4\}$.

Define three operators $L_{k}, M_{k}, \widetilde{L}_{k, \alpha}$ by

$$
\begin{aligned}
& \widehat{L_{k} f}(\xi)=\varphi\left(2^{-k}|\xi|\right) \widehat{f}(\xi), \\
& \widehat{M_{k} f}(\xi)=\frac{\widetilde{\varphi}\left(2^{-k}|\xi|\right)}{m\left(2^{-k} \xi\right)} \widehat{f}(\xi),
\end{aligned}
$$




$$
\widetilde{\widetilde{L}_{k, \alpha} f}(\xi)=\widetilde{\varphi}\left(2^{-k}|\xi|\right)\left(2^{-k}|\xi|\right)^{\alpha} \widehat{f}(\xi) .
$$

These convolution operators make sense for Hilbert-space valued functions.

Below we shall use the following

Lemma 5.1 Let $1<p<\infty$.

(i) For $\left\{f_{k}\right\} \in L^{p}\left(\ell^{2}\right)$

$$
\left\|\sum_{k \in \mathbb{Z}} L_{k} f_{k}\right\|_{p} \leq C_{p}\left\|\left(\sum_{k \in \mathbb{Z}}\left|f_{k}\right|^{2}\right)^{1 / 2}\right\|_{p} .
$$

(ii) $\operatorname{For}\left\{f_{k}\right\} \in L^{p}\left(\ell^{2}\right)$

$$
\left\|\left(\sum_{k \in \mathbb{Z}}\left|M_{k} f_{k}\right|^{2}\right)^{1 / 2}\right\|_{p} \leq C_{p}\left\|\left(\sum_{k \in \mathbb{Z}}\left|f_{k}\right|^{2}\right)^{1 / 2}\right\|_{p} .
$$

(iii) Let $\mathcal{H}$ be a Hilbert space. For $F \in L^{p}(\mathcal{H})$ we have

$$
\left\|\left(\sum_{k \in \mathbb{Z}}\left|\widetilde{L}_{k, \alpha} F\right|_{\mathcal{H}}^{2}\right)^{1 / 2}\right\|_{p} \leq C_{p, \alpha}\|F\|_{L^{p}(\mathcal{H})} .
$$

Proof These are straightforward applications of the standard theory of singular convolution operators for Hilbert-space valued functions, see [1,17].

Proof of (12) Define $L_{k}$ as above and $\widetilde{L}_{k}$ similarly, with $\varphi$ replaced by $\widetilde{\varphi}$. We then have

$$
f=\sum_{k \in \mathbb{Z}} L_{k} f=\sum_{k \in \mathbb{Z}} L_{k} \widetilde{L}_{k} \widetilde{L}_{k} f=\sum_{k \in \mathbb{Z}} L_{k} M_{k} \widetilde{L}_{k} \mathcal{F}^{-1}\left[m\left(2^{-k} \cdot\right) \widehat{f}\right] .
$$

By (i), (ii) of Lemma (5.1) we have

$$
\begin{aligned}
\|f\|_{p} & \lesssim\left\|\left(\sum_{k \in \mathbb{Z}}\left|M_{k} \widetilde{L}_{k} \mathcal{F}^{-1}\left[m\left(2^{-k} \cdot\right) \widehat{f}\right]\right|^{2}\right)^{1 / 2}\right\|_{p} \\
& \leq\left\|\left(\sum_{k}\left|\frac{1}{\left|R_{\varepsilon}\right|} \iint_{R_{\varepsilon}} T_{k}^{s, t} f \mathrm{~d} s \mathrm{~d} t\right|^{2}\right)^{1 / 2}\right\|_{p}
\end{aligned}
$$

where $T_{k}^{s, t}$ is defined by

$$
\begin{aligned}
\widehat{T_{k}^{s, t} f}(\xi) & =\widetilde{\varphi}\left(2^{-k}|\xi|\right)\left(\frac{e^{i s\left(2^{-k} \xi\right)}-1}{s}-\frac{e^{i t\left(2^{-k} \xi\right)}-1}{t}\right)|s-t|^{-\alpha} \widehat{f}(\xi) \\
& =2^{-k} \widetilde{\varphi}\left(2^{-k}|\xi|\right)\left(2^{-k}|\xi|\right)^{\alpha}\left(\frac{e^{i\left(2^{-k} s\right) \xi}-1}{2^{-k} s}-\frac{e^{i\left(2^{-k} t\right) \xi}-1}{2^{-k} t}\right) \frac{\widehat{\mathcal{D}^{-\alpha} f}(\xi)}{\left(2^{-k}|s-t|\right)^{\alpha}} .
\end{aligned}
$$

We apply the Cauchy-Schwarz inequality on $R_{\varepsilon}$ and get

$$
\|f\|_{p} \leq\left\|\left(\sum_{k} \frac{1}{\left|R_{\varepsilon}\right|} \iint_{R_{\varepsilon}}\left|T_{k}^{s, t} f\right|^{2} \mathrm{~d} s \mathrm{~d} t\right)^{1 / 2}\right\|_{p} .
$$


Change variables $s=2^{k} v, t=2^{k} w$ so that the last inequality becomes

$$
\begin{aligned}
& \|f\|_{p} \\
& \quad \lesssim\left\|\left(\sum_{k} \frac{1}{\left|R_{\varepsilon}\right|} \iint_{2^{-k} R_{\varepsilon}}\left|\frac{\widetilde{L}_{k, \alpha}\left[v^{-1} \Delta_{v}\left(\mathcal{D}^{-\alpha} f\right)-w^{-1} \Delta_{w}\left(\mathcal{D}^{-\alpha} f\right)\right]}{|v-w|^{\alpha}}\right|^{2} \mathrm{~d} v \mathrm{~d} w\right)^{1 / 2}\right\|_{p} .
\end{aligned}
$$

We replace for each $k$ the domain of integration $2^{-k} R_{\varepsilon}$ by the entire $\mathbb{R} \times \mathbb{R}$ and then apply part (iii) of Lemma 5.1 (with the Hilbert space $\mathcal{H}=L^{2}(\mathbb{R} \times \mathbb{R})$ ). We thus see that $\|f\|_{p}$ is bounded by (a constant times)

$$
\begin{gathered}
\left|R_{\varepsilon}\right|^{-1 / 2}\left\|\left(\sum_{k} \iint_{\mathbb{R} \times \mathbb{R}} \frac{\left|\tilde{L}_{k, \alpha}\left[v^{-1} \Delta_{v}\left(\mathcal{D}^{-\alpha} f\right)-w^{-1} \Delta_{w}\left(\mathcal{D}^{-\alpha} f\right)\right]\right|^{2}}{|v-w|^{2 \alpha}} \mathrm{d} v \mathrm{~d} w\right)^{1 / 2}\right\|_{p} \\
\leq C_{\varepsilon, p, \alpha}\left\|\left(\iint_{\mathbb{R} \times \mathbb{R}} \frac{\left|v^{-1} \Delta_{v}\left(\mathcal{D}^{-\alpha} f\right)-w^{-1} \Delta_{w}\left(\mathcal{D}^{-\alpha} f\right)\right|^{2}}{|v-w|^{2 \alpha}} \mathrm{d} v \mathrm{~d} w\right)^{1 / 2}\right\|_{p}
\end{gathered}
$$

which completes the proof of (12).

\section{$6 L^{2}$ bounds}

As mentioned before the equivalence

$$
\left\|S_{\alpha}\left(\mathcal{D}^{-\alpha} f\right)\right\|_{2}=c_{\alpha}\|f\|_{2}
$$

has been proved for $\alpha=1$ in [11]; a straightforward modification of the proof also applies to the case $\alpha \in(1 / 2,3 / 2)$. In this section we further break up $S_{\alpha}\left(\mathcal{D}^{-\alpha} f\right)$ and obtain improved bounds for the pieces, which are useful for the proof of Theorems 1.1 and 1.2.

Let $\mathcal{H}$ be the Hilbert space of square-integrable functions on $\mathbb{R} \times \mathbb{R}$. Fix $\alpha \in(1 / 2,3 / 2)$. We define a convolution operator $T$ mapping Schwartz functions on $\mathbb{R}$ to $\mathcal{H}$-valued functions on the real line, by

$$
T f(x, s, t)=\frac{s^{-1} \Delta_{s} \mathcal{D}^{-\alpha} f(x)-t^{-1} \Delta_{t} \mathcal{D}^{-\alpha} f(x)}{|s-t|^{\alpha}} \text { for }|s| \geq|t|,
$$

and

$$
T f(x, s, t)=0 \text { for }|s|<|t| .
$$

The inequality $\left\|S_{\alpha}\left(\mathcal{D}^{-\alpha} f\right)\right\|_{p} \lesssim\|f\|_{p}$ holds for all Schwartz functions $f$ if and only if $T$ maps $L^{p}(\mathbb{R})$ to $L^{p}(\mathbb{R} ; \mathcal{H})$.

For the estimates below we may assume that $f$ is a Schwartz function whose Fourier transform is compactly supported in $\mathbb{R} \backslash\{0\}$.

We introduce finer decompositions by dividing up the $(s, t)$ parameter set. For $n, l \in \mathbb{Z}$, $l \leq n-2$, set

$$
\begin{aligned}
\mathcal{V}_{k}^{n, l}= & \left\{(s, t): 2^{-k+n}<|s| \leq 2^{-k+n+1}, 2^{-k+n-2}<|t| \leq|s|,\right. \\
& \left.2^{-k+l-1}<|s-t| \leq 2^{-k+l}\right\},
\end{aligned}
$$

and note that

$$
\mathcal{V}_{k}^{n, l}=\emptyset \text { for } l \geq n+3
$$


Also for $\ell \in \mathbb{Z}, \ell \leq n-2$, let

$$
\mathcal{W}_{k}^{n, \ell}=\left\{(s, t): 2^{-k+n}<|s| \leq 2^{-k+n+1}, 2^{-k+\ell-1}<|t| \leq 2^{-k+\ell}\right\} .
$$

Then, for every $k \in \mathbb{Z}$,

$$
\sum_{n \in \mathbb{Z}}\left(\sum_{l=-\infty}^{n+2} \mathbb{1}_{\mathcal{V}_{k}^{n, l}}(s, t)+\sum_{\ell=-\infty}^{n-2} \mathbb{1}_{\mathcal{W}_{k}^{n . \ell}}(s, t)\right)= \begin{cases}1 & \text { if }|t| \leq|s|, \\ 0 & \text { if }|t|>|s| .\end{cases}
$$

We also observe

$$
\mathcal{V}_{k}^{n, l}=2^{-k} \mathcal{V}_{0}^{n, l}, \quad \mathcal{W}_{k}^{n, \ell}=2^{-k} \mathcal{W}_{0}^{n, \ell}
$$

In what follows we denote by $\psi$ a real valued Schwartz function so that $\widehat{\psi}(\xi) \neq 0$ for $\frac{1}{4} \leq|\xi| \leq 4$ and $\widehat{\psi}$ vanishes to order 100 at the origin. We may choose $\psi$ so that

$$
\operatorname{supp}(\psi) \subset\{x:|x| \leq 1 / 2\}
$$

We remark that this assumption is not needed in the present section, nor in the proof of Theorem 1.1 discussed in Sect. 8.2. However it is quite convenient in the proof of the endpoint bound of Theorem 1.2.

Set $\psi_{k}=2^{k} \psi\left(2^{k}\right.$.). Define an operator $P_{k}$ by

$$
P_{k} f=\psi_{k} * f \text {. }
$$

We introduce a decomposition of the operator $T$. Let $\varphi \in C_{c}^{\infty}$ supported in $\{\xi: 1 / 2<|\xi|<$ $2\}$ so that $\sum_{k \in \mathbb{Z}} \varphi\left(2^{-k} \xi\right)=1$ for all $\xi \neq 0$. We then decompose

$$
\begin{aligned}
& \widehat{T f}(\xi, s, t) \\
& =\sum_{k \in \mathbb{Z}} \widehat{\psi}\left(2^{-k} \xi\right)^{2} \frac{\varphi\left(2^{-k} \xi\right)}{\left(2^{-k}|\xi|\right)^{\alpha}\left(\widehat{\psi}\left(2^{-k} \xi\right)\right)^{2}} \frac{1}{2^{k \alpha}|s-t|^{\alpha}}\left(\frac{e^{i \xi s}-1}{s}-\frac{e^{i \xi t}-1}{t}\right) \widehat{f}(\xi)
\end{aligned}
$$

and hence

$$
T f(x, s, t)=\sum_{k \in \mathbb{Z}} P_{k} T_{k} L_{k} f(x, s, t)
$$

where

$$
\widehat{L_{k} f}(\xi)=\frac{\varphi\left(2^{-k} \xi\right)}{\left(2^{-k}|\xi|\right)^{\alpha}\left(\widehat{\psi}\left(2^{-k} \xi\right)\right)^{2}} \widehat{f}(\xi)
$$

and

$$
T_{k} f(x, s, t)=\frac{1}{2^{k \alpha}|s-t|^{\alpha}}\left(\frac{P_{k} f(x+s)-P_{k} f(x)}{s}-\frac{P_{k} f(x+t)-P_{k} f(x)}{t}\right)
$$

when $|t| \leq|s|$ (and $T_{k} f(x, s, t)=0$ otherwise). We also set, for $|t|<|s|$,

$$
\begin{aligned}
& T_{k, 1} f(x, s, t)=\frac{1}{2^{k \alpha}|s-t|^{\alpha}} \frac{P_{k} f(x+s)-P_{k} f(x)}{s}, \\
& T_{k, 2} f(x, s, t)=\frac{1}{2^{k \alpha}|s-t|^{\alpha}} \frac{P_{k} f(x+t)-P_{k} f(x)}{t}
\end{aligned}
$$

so that $T_{k}=T_{k, 1}-T_{k, 2}$.

We shall repeatedly use the following scaling lemma. 
Lemma 6.1 Let $g$ be a Schwartz function on $\mathbb{R}$. For $k \in \mathbb{Z}$ and $\Omega \subset \mathbb{R}^{2}$,

$$
\left(\iint_{\Omega}\left|T_{k} g(x, s, t)\right|^{2} \mathrm{~d} s \mathrm{~d} t\right)^{1 / 2}=\left(\iint_{2^{k} \Omega}\left|T_{0}\left[g\left(2^{-k} \cdot\right)\right]\left(2^{k} x, v, w\right)\right|^{2} \mathrm{~d} v \mathrm{~d} w\right)^{1 / 2}
$$

Proof The left hand side is equal to

$$
\begin{aligned}
& \left(\iint_{\Omega}\left|\frac{P_{k} g(x+s)-P_{k} g(x)}{s}-\frac{P_{k} g(x+t)-P_{k} g(x)}{t}\right|^{2} \frac{\mathrm{d} s \mathrm{~d} t}{2^{2 k \alpha}|s-t|^{2 \alpha}}\right)^{1 / 2} \\
& \quad=\left(\iint_{2^{k} \Omega}\left|\frac{P_{k} g\left(x+2^{-k} v\right)-P_{k} g(x)}{v}-\frac{P_{k} g\left(x+2^{-k} w\right)-P_{k} g(x)}{w}\right|^{2} \frac{\mathrm{d} v \mathrm{~d} w}{|v-w|^{2 \alpha}}\right)^{1 / 2}
\end{aligned}
$$

and the assertion follows from $P_{k} g(x)=P_{0}\left[g\left(2^{-k} \cdot\right)\right]\left(2^{k} x\right)$.

Our proof of $L^{2}$ boundedness involves the following elementary estimates.

Lemma 6.2 Let $a \geq b>0$. Then,

(i)

$$
\left(\iint_{\substack{|\sigma| \approx|\tau| \approx a \\|\sigma-\tau| \approx b}}\left|\frac{e^{i \sigma}-1}{\sigma}-\frac{e^{i \tau}-1}{\tau}\right|^{2} \frac{\mathrm{d} \sigma \mathrm{d} \tau}{|\sigma-\tau|^{2 \alpha}}\right)^{1 / 2} \lesssim \begin{cases}a^{\frac{1}{2}} b^{\frac{3}{2}-\alpha} & \text { if } a \leq 1, \\ a^{-\frac{1}{2}} b^{\frac{3}{2}-\alpha} & \text { if } b \leq 1 \leq a, \\ a^{-\frac{1}{2}} b^{\frac{1}{2}-\alpha} & \text { if } 1 \leq b \leq a .\end{cases}
$$

(ii) Let $a \geq \gamma$ and $\Omega_{a, \gamma}:=\{(\sigma, \tau):|\tau| \leq|\sigma| / 2,|\tau| \approx \gamma,|\sigma| \approx a\}$. Then, for $a \geq 1$

$$
\begin{aligned}
& \left(\iint_{\Omega_{a, \gamma}}\left|\frac{e^{i \sigma}-1}{\sigma}\right|^{2} \frac{\mathrm{d} \sigma \mathrm{d} \tau}{|\sigma-\tau|^{2 \alpha}}\right)^{1 / 2} \lesssim \gamma^{\frac{1}{2}} a^{-\alpha} \min \left\{a^{\frac{1}{2}}, a^{-\frac{1}{2}}\right\}, \\
& \left(\iint_{\Omega_{a, \gamma}}\left|\frac{e^{i \tau}-1}{\tau}\right|^{2} \frac{\mathrm{d} \sigma \mathrm{d} \tau}{|\sigma-\tau|^{2 \alpha}}\right)^{1 / 2} \lesssim a^{\frac{1}{2}-\alpha} \min \left\{\gamma^{\frac{1}{2}}, \gamma^{-\frac{1}{2}}\right\} .
\end{aligned}
$$

Moreover, if $a \leq 1$ then

$$
\left(\iint_{\Omega_{a, \gamma}}\left|\frac{e^{i \sigma}-1}{\sigma}-\frac{e^{i \tau}-1}{\tau}\right|^{2} \frac{\mathrm{d} \sigma \mathrm{d} \tau}{|\sigma-\tau|^{2 \alpha}}\right)^{1 / 2} \lesssim \gamma^{\frac{1}{2}} a^{\frac{3}{2}-\alpha} .
$$

Proof This follows readily from

$$
\left|\frac{e^{i \sigma}-1}{\sigma}-\frac{e^{i \tau}-1}{\tau}\right| \leq C \frac{|\sigma-\tau|}{1+|\sigma|+|\tau|}, \quad|\sigma-\tau| \leq 10 .
$$

and

$$
\left|\frac{e^{i r}-1}{r}\right| \leq C \min \left\{1, r^{-1}\right\}
$$

Proposition 6.3 Let $1 / 2<\alpha<3 / 2$ and let $\mathcal{V}_{k}^{n, l}, \mathcal{W}_{k}^{n, \ell}$ be as in (15), (17). Then, the following estimates hold. 
(i)

$$
\begin{gathered}
\left\|\left(\iint\left|\sum_{\substack{k \in \mathbb{Z}: \\
(s, t) \in \mathcal{V}_{k}^{n, l}}} P_{k} T_{k} f_{k}(\cdot, s, t)\right|^{2} \mathrm{~d} s \mathrm{~d} t\right)^{1 / 2}\right\| c_{n, l}\left\|\left(\sum_{k}\left|f_{k}\right|^{2}\right)^{1 / 2}\right\|_{2}, \\
\text { with }_{n, l}= \begin{cases}2^{\frac{n}{2}} 2^{l\left(\frac{3}{2}-\alpha\right)} & \text { if } n \leq 0, l \leq n+2 \\
2^{-n / 2} 2^{l\left(\frac{3}{2}-\alpha\right)} & \text { if } n \geq 0, l \leq 2 \\
2^{-n / 2} 2^{-l\left(\alpha-\frac{1}{2}\right)} & \text { if } n \geq 0,0 \leq l \leq n+2 \\
0 & \text { if } l>n+2\end{cases}
\end{gathered}
$$

(ii) Let $n \geq 0$ and $\ell \leq n-2$. Then,

$$
\begin{aligned}
& \left\|\left(\iint\left|\sum_{\substack{k \in \mathbb{Z}: \\
(s, t) \in \mathcal{W}_{k}^{n, \ell}}} P_{k} T_{k, 1} f_{k}(\cdot, s, t)\right|^{2} \mathrm{~d} s \mathrm{~d} t\right)^{1 / 2}\right\|_{2} \\
& \lesssim 2^{\ell / 2} 2^{-n \alpha} \min \left\{2^{-n / 2}, 2^{n / 2}\right\}\left\|\left(\sum_{k}\left|f_{k}\right|^{2}\right)^{1 / 2}\right\|_{2}
\end{aligned}
$$

and

$$
\begin{aligned}
& \left\|\left(\iint\left|\sum_{\substack{k \in \mathbb{Z}: \\
(s, t) \in \mathcal{W}_{k}^{n, \ell}}} P_{k} T_{k, 2} f_{k}(\cdot, s, t)\right|^{2} \mathrm{~d} s \mathrm{~d} t\right)^{1 / 2}\right\|_{2}\left\|_{2}^{1 / 2}\right\|_{k} . \\
& \lesssim 2^{-n\left(\alpha-\frac{1}{2}\right)} \min \left\{2^{-\ell / 2}, 2^{\ell / 2}\right\} \|\left(\sum_{k}\left|f_{k}\right|^{2} \|_{2} .\right.
\end{aligned}
$$

Moreover, for $n \leq 0$,

$$
\begin{gathered}
\left\|\left(\iint\left|\sum_{\substack{k \in \mathbb{Z}: \\
(s, t) \in \mathcal{W}_{k}^{n, \ell}}} P_{k} T_{k} f_{k}(\cdot, s, t)\right|^{2} \mathrm{~d} s \mathrm{~d} t\right)^{1 / 2}\right\|_{2} \\
\lesssim 2^{n\left(\frac{3}{2}-\alpha\right)} 2^{\ell / 2}\left\|\left(\sum_{k}\left|f_{k}\right|^{2}\right)^{1 / 2}\right\|_{2} .
\end{gathered}
$$

Proof Note that for fixed $n, l$ the sets $\mathcal{V}_{k}^{n, l}, k \in \mathbb{Z}$ are disjoint and, similarly, for fixed $n, \ell$ the sets $\mathcal{W}_{k}^{n, \ell}, k \in \mathbb{Z}$ are disjoint. Hence, $\iint\left|\sum_{k} \cdots\right|^{2} \mathrm{~d} s \mathrm{~d} t=\iint \sum_{k}|\cdots|^{2} \mathrm{~d} s \mathrm{~d} t$. Thus, if one then interchanges sums and integrals and uses the uniform $L^{2}$ boundedness of the operators $P_{k}$ one can reduce the proofs to showing uniform estimates for the individual operators $T_{k}$ (or $T_{k, 1}, T_{k, 2}$ ), involving the sets $\mathcal{V}_{k}^{n, l}, \mathcal{W}_{k}^{m, \ell}$. By Lemma 6.1 this is reduced to use estimates 
for the operator $T_{0}$ (or $T_{0,1}, T_{0,2}$ ), involving localizations to the sets $\mathcal{V}_{0}^{n, l}, \mathcal{W}_{0}^{m, \ell}$. Let

$$
m(\xi, s)=\widehat{\psi}(\xi) s^{-1}\left(e^{i s \xi}-1\right) .
$$

All estimates in Proposition 6.3 follow via Plancherel's theorem from the following set (29) of inequalities. First, with $c_{n, l}$ as in (28a),

$$
\sup _{\xi}\left(\iint_{\mathcal{V}_{0}^{n, l}} \frac{|m(\xi, s)-m(\xi, t)|^{2}}{|s-t|^{2 \alpha}} \mathrm{d} s \mathrm{~d} t\right)^{1 / 2} \lesssim c_{n, l} .
$$

Next,

$$
\begin{aligned}
& \sup _{\xi}\left(\iint_{\mathcal{W}_{0}^{n, \ell}} \frac{|m(\xi, s)|^{2}}{|s-t|^{2 \alpha}} \mathrm{d} s \mathrm{~d} t\right)^{1 / 2} \lesssim 2^{\ell / 2} 2^{-n \alpha} \min \left\{2^{-n / 2}, 2^{n / 2}\right\}, \\
& \sup _{\xi}\left(\iint_{\mathcal{W}_{0}^{n, \ell}} \frac{|m(\xi, t)|^{2}}{|s-t|^{2 \alpha}} \mathrm{d} s \mathrm{~d} t\right)^{1 / 2} \lesssim 2^{-n\left(\alpha-\frac{1}{2}\right)} \min \left\{2^{-\ell / 2}, 2^{\ell / 2}\right\},
\end{aligned}
$$

and (for $\ell+2 \leq n \leq 0)$

$$
\sup _{\xi}\left(\iint_{\mathcal{W}_{0}^{n, \ell}} \frac{|m(\xi, s)-m(\xi, t)|^{2}}{|s-t|^{2 \alpha}} \mathrm{d} s \mathrm{~d} t\right)^{1 / 2} \lesssim 2^{n\left(\frac{3}{2}-\alpha\right)} 2^{\frac{\ell}{2}} .
$$

We want to deduce (29) from Lemma 6.2. In view of the crucial cancellation property of $\psi$ we have

$$
|\widehat{\psi}(\xi)| \leq C_{N} \frac{|\xi|^{2}}{(1+|\xi|)^{N}}
$$

for all $N$. Now by a change of variables

$$
\begin{aligned}
& \left(\iint_{\Omega} \frac{|m(\xi, s)-m(\xi, t)|^{2}}{|s-t|^{2 \alpha}} \mathrm{d} s \mathrm{~d} t\right)^{1 / 2} \\
& \quad=|\xi|^{\alpha}|\widehat{\psi}(\xi)|\left(\iint_{|\xi| \Omega}\left|\frac{e^{i \sigma}-1}{\sigma}-\frac{e^{i \tau}-1}{\tau}\right|^{2} \frac{\mathrm{d} \sigma \mathrm{d} \tau}{|\sigma-\tau|^{2 \alpha}}\right)^{1 / 2} .
\end{aligned}
$$

Hence, by Lemma 6.2 and (30),

$$
\begin{aligned}
& \left(\iint_{\mathcal{V}_{0}^{n, l}} \frac{|m(\xi, s)-m(\xi, t)|^{2}}{|s-t|^{2 \alpha}} \mathrm{d} s \mathrm{~d} t\right)^{1 / 2} \\
& \lesssim \frac{|\xi|^{\alpha+2}}{(1+|\xi|)^{N}} \cdot \begin{cases}\left(2^{n}|\xi|\right)^{1 / 2}\left(2^{l}|\xi|\right)^{3 / 2-\alpha} & \text { if } 2^{n}|\xi| \leq 1, l \leq n+2 \\
\left(2^{n}|\xi|\right)^{-1 / 2}\left(2^{l}|\xi|\right)^{3 / 2-\alpha} & \text { if } 2^{n}|\xi| \geq 1,2^{l}|\xi| \leq 1 \\
\left(2^{n}|\xi|\right)^{-1 / 2}\left(2^{l}|\xi|\right)^{-\alpha+1 / 2} & \text { if } 2^{n}|\xi| \geq 1,0 \leq l \leq n+2\end{cases}
\end{aligned}
$$

which implies (29a). The estimates (29b), (29c), (29d) follow in a similar way from Lemma 6.2 and (30).

We finally note for further reference that summing the various estimates in Proposition 6.3 together with an application of the Littlewood-Paley inequality (in $L^{2}$ ) yields the bound $\|\mathcal{T} F\|_{2} \lesssim\|F\|_{L^{2}(\mathcal{H})}$. 


\section{The $H^{1} \rightarrow L^{1, \infty}$ bound}

We shall follow the method outlined in [15] which has its root in work by Christ [5]. We use a variant of the atomic decomposition which also takes our operator $T$ into account (by using the decomposition (21) and incorporating the Riesz potential operator in the atoms). The approach here is based on the square-function characterization by Chang and Fefferman [4] (in the one-parameter dilation setting). See also [14] for an early application to endpoint estimates, and [15] for many more references.

\subsection{Preliminaries}

Let $P_{k}, T_{k}, L_{k}$ as in (20), (23), (22). We plan to use the decomposition (21). We consider the nontangential version of the Peetre maximal operators

$$
\mathfrak{M}_{k} f(x)=\sup _{|h| \leq 2^{-k}}\left|L_{k} f(x+h)\right|
$$

and the square function defined by

$$
\mathfrak{S} f(x)=\left(\sum_{k \in \mathbb{Z}}\left|\mathfrak{M}_{k} f(x)\right|^{2}\right)^{1 / 2} .
$$

Then, (Peetre [12])

$$
\|\mathfrak{S} f\|_{L^{1}} \lesssim\|f\|_{H^{1}}
$$

Let $\mathfrak{J}_{k}$ be the set of dyadic intervals of length $2^{-k}$ (i.e., each interval is of the form $\left[n 2^{-k},(n+\right.$ 1) $2^{-k}$ ) for some $n \in \mathbb{Z}$ ). For $\mu \in \mathbb{Z}$ let

$$
\mathcal{O}_{\mu}=\left\{x:|\mathfrak{S} f(x)|>2^{\mu}\right\}
$$

and let $\mathfrak{J}_{k}^{\mu}$ be the set of dyadic intervals of length $2^{-k}$ with the property that

$$
\left|J \cap \mathcal{O}_{\mu}\right| \geq|J| / 2 \text { and }\left|J \cap \mathcal{O}_{\mu+1}\right|<|J| / 2 .
$$

Clearly if $\mathfrak{S} f \in L^{1}$ then every dyadic interval belongs to exactly one of the sets $\mathfrak{J}_{k}^{\mu}$. We then have ([4])

$$
\sum_{k \in \mathbb{Z}} \sum_{J \in \mathfrak{J}_{k}^{\mu}}\left\|\mathbb{1}_{J} L_{k} f\right\|_{2}^{2} \lesssim 2^{2 \mu} \operatorname{meas}\left(\mathcal{O}_{\mu}\right)
$$

For completeness we include the argument for (34). The relevant fact is that $\left|L_{k} f(x)\right| \leq$ $\mathfrak{M}_{k} f(z)$ for all $x, z \in J$, for each $J \in \mathfrak{J}_{k}$. Let

$$
\mathcal{O}_{\mu}^{*}=\left\{x: M_{H L} \mathbb{1}_{\mathcal{O}_{\mu}}>10^{-1}\right\}
$$

where $M_{H L}$ stands for the Hardy-Littlewood maximal operator. Then,

$$
\operatorname{meas}\left(\mathcal{O}_{\mu}^{*}\right) \lesssim \operatorname{meas}\left(\mathcal{O}_{\mu}\right)
$$

and we have $\cup_{k} \cup_{J \in \mathfrak{J}_{k}^{\mu}} J \subset \mathcal{O}_{\mu}^{*}$. Now

$$
\begin{aligned}
& \sum_{k \in \mathbb{Z}} \sum_{J \in \mathfrak{J}_{k}^{\mu}}\left\|\mathbb{1}_{J} L_{k} f\right\|_{2}^{2} \leq \sum_{k \in \mathbb{Z}} \sum_{J \in \mathfrak{J}_{k}^{\mu}} 2 \int_{J \backslash \mathcal{O}^{\mu+1}}\left|\mathfrak{M}_{k} f(x)\right|^{2} \mathrm{~d} x \\
& \quad \leq 2 \int_{\mathcal{O}_{\mu}^{*} \backslash \mathcal{O}^{\mu+1}} \sum_{k \in \mathbb{Z}}\left|\mathfrak{M}_{k} f(x)\right|^{2} \mathrm{~d} x \leq 2^{2 \mu+2} \operatorname{meas}\left(\mathcal{O}_{\mu}^{*}\right) \leq C 2^{2 \mu} \operatorname{meas}\left(\mathcal{O}_{\mu}\right)
\end{aligned}
$$


which establishes (34).

Now we assign to each dyadic interval $J$ another dyadic interval $I(J)$ containing $J$. If $J \in \mathfrak{J}_{k}^{\mu}$ then clearly $J \subset \mathcal{O}_{\mu}^{*}$. Let $I(J)$ be the maximal dyadic interval containing $J$ which is contained in $\mathcal{O}_{\mu}^{*}$. Set

$$
b_{k}^{\mu, I}(x)=\sum_{\substack{J \in \mathfrak{J}_{k}^{\mu}: \\ I(J)=I}} L_{k} f(x) \mathbb{1}_{J}(x)
$$

We write $L(I)=L$ if the length of a dyadic interval $I$ is $2^{L}$. Also we let $\mathcal{I}^{\mu}$ be the collection of all dyadic intervals which are maximal and contained in $\mathcal{O}_{\mu}^{*}$. By the maximality condition the intervals in $\mathcal{I}^{\mu}$ have disjoint interior. For future reference we note that if $J \in \mathfrak{J}_{k}^{\mu}$ and $I(J)=I$ then $L(I)+k \geq 0$.

Set, for $I \in \mathcal{I}^{\mu}$,

$$
\gamma_{\mu, I}:=\left(\sum_{\substack{k: \\ k+L(I) \geq 0}} \sum_{J \in \mathfrak{J}_{\mu}^{k}}\left\|\mathbb{1}_{J} L_{k} f\right\|_{2}^{2}\right)^{1 / 2} .
$$

We have

$$
\begin{aligned}
\sum_{I \in \mathcal{I}^{\mu}}|I|^{1 / 2} \gamma_{\mu, I} & \leq\left(\sum_{I \in \mathcal{I}^{\mu}}|I|\right)^{1 / 2}\left(\sum_{I \in \mathcal{I}^{\mu}} \gamma_{\mu, I}^{2}\right)^{1 / 2} \\
& \lesssim\left|\mathcal{O}_{\mu}^{*}\right|^{1 / 2}\left(2^{2 \mu}\left|\mathcal{O}_{\mu}\right|\right)^{1 / 2} \lesssim 2^{\mu}\left|\mathcal{O}_{\mu}\right|
\end{aligned}
$$

and hence

$$
\sum_{\mu \in \mathbb{Z}} \sum_{I \in \mathcal{I}^{\mu}}|I|^{1 / 2} \gamma_{\mu, I} \lesssim \sum_{\mu \in \mathbb{Z}} 2^{\mu}\left|\mathcal{O}_{\mu}\right| \lesssim\|\mathfrak{S} f\|_{1} \lesssim\|f\|_{H^{1}}
$$

which is equivalent to

$$
\sum_{\mu \in \mathbb{Z}} \sum_{I \in \mathcal{I}^{\mu}}|I|^{1 / 2}\left(\sum_{k \in \mathbb{Z}}\left\|b_{k}^{\mu, I}\right\|_{2}^{2}\right)^{1 / 2} \lesssim\|f\|_{H^{1}} .
$$

\subsection{Proof of the $H^{1} \rightarrow L^{1, \infty}$ inequality}

Fix $\lambda>0$. We claim that

$$
\operatorname{meas}\left(\left\{x \in \mathbb{R}:|T f(x, \cdot, \cdot)|_{L^{2}\left(\mathbb{R}^{2}\right)}>10 \lambda\right\}\right) \lesssim \lambda^{-1} \sum_{\mu \in \mathbb{Z}} \sum_{I \in \mathcal{I}^{\mu}}|I|^{1 / 2} \gamma_{\mu, I}
$$

which implies the desired bound, by (36).

The first step is the definition of an exceptional set $\mathcal{E}$. Given any $I, \mu$ with $\mu \in \mathbb{Z}, I \in \mathfrak{J}^{\mu}$, we assign an integer $\kappa(\mu, I)$ (depending on $\lambda$ ), defined as

$$
\kappa(\mu, I)=\max \{L(I), \tilde{\varkappa}(\mu, I)\}
$$

where the "stopping time" $\tilde{\varkappa}(\mu, I)$ is given by

$$
\tilde{\mathcal{\varkappa}}(\mu, I)=\inf \left\{r \in \mathbb{Z}: 2^{r} \geq \lambda^{-1}|I|^{1 / 2} \gamma_{\mu, I}\right\} .
$$


For any $I, \mu$ satisfying $L(I)<\kappa(\mu, I)$ let $\mathcal{E}_{\mu, I}$ be the interval of length $2^{\kappa(\mu, I)+5}$, concentric with $I$ and let

$$
\mathcal{E}=\bigcup_{\substack{\mu, I: I \in \mathcal{I}^{\mu} \\ L(I)<\kappa(\mu, I)}} \mathcal{E}_{\mu, I}
$$

For any $I$ with $\kappa(\mu, I)>L(I)$, we have $2^{\kappa(\mu, I)-1} \leq \lambda^{-1}|I|^{1 / 2} \gamma_{\mu, I}$. Thus

$$
\operatorname{meas}(\mathcal{E}) \lesssim \sum_{I, \mu: L(I)<\kappa(\mu, I)} 2^{\kappa(\mu, I)} \lesssim \sum_{I, \mu} \lambda^{-1}|I|^{1 / 2} \gamma_{\mu, I} \lesssim \lambda^{-1}\|f\|_{H^{1}}
$$

Hence, in order to prove (38) we only need to show

$$
\operatorname{meas}\left(x \in \mathcal{E}^{\complement}:|T f(x, \cdot, \cdot)|_{L^{2}\left(\mathbb{R}^{2}\right)}>10 \lambda\right\} \lesssim \lambda^{-1} \sum_{\mu, I}|I|^{1 / 2} \gamma_{\mu, I} .
$$

By Minkowski’s inequality we have

$$
\begin{aligned}
|T f(x, \cdot, \cdot)|_{L^{2}\left(\mathbb{R}^{2}\right)} & =\left(\iint\left|\sum_{\mu, I} \sum_{-k \leq L(I)} P_{k} T_{k} b_{k}^{\mu, I}(x, s, t)\right|^{2} \mathrm{~d} s \mathrm{~d} t\right)^{1 / 2} \\
& \leq \sum_{i=1}^{4} U_{i}(x)+\mathcal{V}_{\mathcal{E}}(x)+\mathcal{W}_{2, \mathcal{E}}(x)+V_{*}(x)+W_{2, *}(x)+W_{1}(x)
\end{aligned}
$$

where

$$
\begin{aligned}
U_{1}(x)= & \left(\left.\iint\left|\sum_{\substack{n \\
\ell \leq n+2}} \sum_{k:(s, t) \in \mathcal{V}_{k}^{n, l}} \sum_{\substack{\mu, I: \\
I \in \mathcal{I}^{\mu} \\
-k+n \leq L(I)}} P_{k} T_{k} b_{k}^{\mu, I}(x, s, t)\right|^{2} \mathrm{~d} s \mathrm{~d} t\right|^{1 / 2},\right. \\
U_{2}(x)= & \left(\left.\iint\left|\sum_{\substack{n \leq 2 \\
\ell \leq n-2}} \sum_{k:(s, t) \in \mathcal{W}_{k}^{n, \ell}} \sum_{\substack{\mu, I: I \in \mathcal{I}^{\mu} \\
-k \leq L(I)}} P_{k} T_{k} b_{k}^{\mu, I}(x, s, t)\right|^{2} \mathrm{~d} s \mathrm{~d} t\right|^{1 / 2},\right. \\
U_{3}(x)= & \left.\left.\iint \sum_{\substack{n \geq 2 \\
\ell \leq n-2}} \sum_{\substack{\mu:(s, t) \in \mathcal{W}_{k}^{n, \ell} \\
-k+I: I \in \mathcal{I}^{\mu}}} P_{k} T_{k, 1} b_{k}^{\mu, I}(x, s, t)\right|^{2} \mathrm{~d} s \mathrm{~d} t\right)^{1 / 2}, \\
U_{4}(x)= & \left(\iint\left|\sum_{\substack{n \geq 2 \\
\ell \leq n-2}} \sum_{\substack{k:(s, t) \in \mathcal{W}_{k}^{n, \ell} \\
-k, I: I \in \mathcal{I}^{\mu}}} P_{k} T_{k, 2} b_{k}^{\mu, I}(x, s, t)\right|^{2} \mathrm{~d} s \mathrm{~d} t\right)^{1 / 2},
\end{aligned}
$$


and

$$
\begin{aligned}
& V_{\mathcal{E}}(x)=\left(\iint\left|\sum_{\substack{n, l: \\
n \geq \max \{0, l-2\}}} \sum_{\substack { k:(s, t) \in \mathcal{V}_{k}^{n, l} \\
\begin{subarray}{c}{\mu, I: \\
I \in \mathcal{I}^{\mu} \\
L(I)<-k+n \leq \kappa(\mu, I){ k : ( s , t ) \in \mathcal { V } _ { k } ^ { n , l } \\
\begin{subarray} { c } { \mu , I : \\
I \in \mathcal { I } ^ { \mu } \\
L ( I ) < - k + n \leq \kappa ( \mu , I ) } }\end{subarray}} P_{k} T_{k} b_{k}^{\mu, I}(x, s, t)\right|^{2} \mathrm{~d} s \mathrm{~d} t\right)^{1 / 2},
\end{aligned}
$$

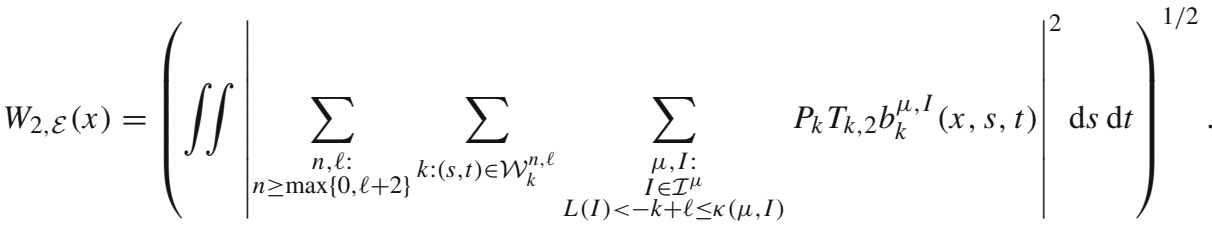

Furthermore

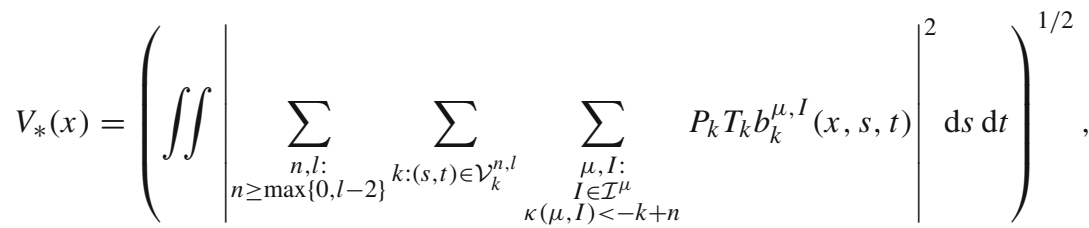

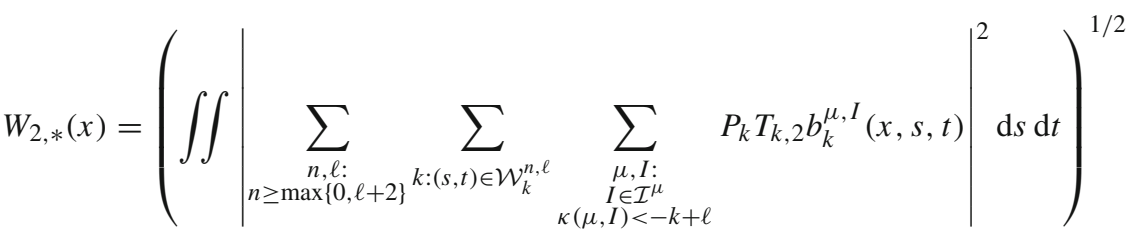

and finally

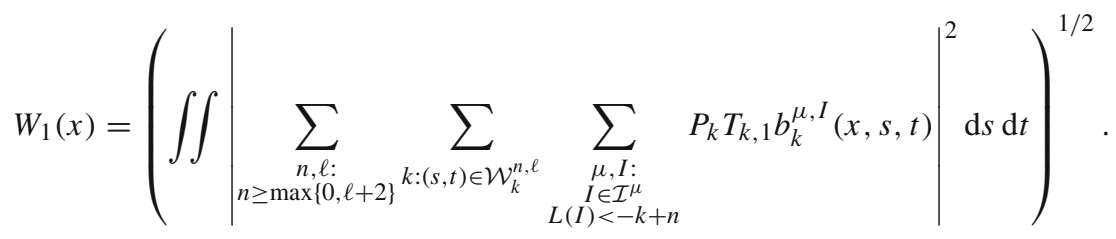

The quantity on the left hand side of (41) is not greater than

$$
\begin{aligned}
& \sum_{i=1}^{4} \operatorname{meas}\left(x \in \mathcal{E}^{\complement}: U_{i}(x)>\lambda\right\}+\operatorname{meas}\left(x \in \mathcal{E}^{\complement}: W_{1}(x)>\lambda\right\} \\
& \quad+\operatorname{meas}\left(x \in \mathcal{E}^{\complement}: V_{*}(x)>\lambda\right\}+\operatorname{meas}\left(x \in \mathcal{E}^{\complement}: W_{2, *}(x)>\lambda\right\} \\
& \quad+\operatorname{meas}\left(x \in \mathcal{E}^{\complement}: V_{\mathcal{E}}(x)>\lambda\right\}+\operatorname{meas}\left(x \in \mathcal{E}^{\complement}: W_{2, \mathcal{E}}(x)>\lambda\right\} .
\end{aligned}
$$


The terms $V_{\mathcal{E}}$ and $W_{2, \mathcal{E}}$ are supported in $\mathcal{E}$ and are thus irrelevant for the estimate (41). Thus (41) follows, by Tshebyshev's inequality, from the bounds

$$
\begin{aligned}
\sum_{i=1}^{4}\left\|U_{i}\right\|_{1} & \lesssim \sum_{\mu, I}|I|^{1 / 2} \gamma_{\mu, I}, \\
\left\|V_{*}\right\|_{2}^{2} & \lesssim \lambda \sum_{\mu, I}|I|^{1 / 2} \gamma_{\mu, I}, \\
\left\|W_{2, *}\right\|_{2}^{2} & \lesssim \lambda \sum_{\mu, I}|I|^{1 / 2} \gamma_{\mu, I}, \\
\left\|W_{1}\right\|_{1} & \lesssim \sum_{\mu, I}|I|^{1 / 2} \gamma_{\mu, I} .
\end{aligned}
$$

\section{Proof of (47)}

For $(s, t) \in \mathcal{V}_{k}^{n, l}$ and $-k+n \leq L(I)$ the function $x \mapsto P_{k} T_{k} b_{k}^{\mu, I}(x, s, t)$ is supported in a tenfold expansion $I^{*}$ of $I$. We use Minkowski's inequality for the $n, l, \mu, I$ sums, and then Cauchy-Schwarz on $I^{*}$ to get

$$
\begin{aligned}
\left\|U_{1}\right\|_{1} \leq & \sum_{\substack{n \\
l \leq n+2}} \sum_{\substack{\mu, I: \\
I \in \mathcal{I}^{\mu}}}\left\|\left(\left.\iint \sum_{\substack{k:(s, t) \in \mathcal{V}_{k}^{n, l} \\
-k+n \leq L(I)}} P_{k} T_{k} b_{k}^{\mu, I}(\cdot, s, t)\right|^{2} \mathrm{~d} s \mathrm{~d} t\right)^{1 / 2}\right\|_{1}^{1 / 2} \\
& \lesssim \sum_{\substack{n \\
l \leq n+2}} \sum_{\substack{\mu, I: \\
I \in \mathcal{I}^{\mu}}}|I|^{1 / 2}\left(\iint \sum_{\substack{k:(s, t) \in \mathcal{V}_{k}^{n, l} \\
-k+n \leq L(I)}} P_{k} T_{k} b_{k}^{\mu, I}(\cdot, s, t) \|^{2} \mathrm{~d} s \mathrm{~d} t\right)^{\|} .
\end{aligned}
$$

Denote by $c_{n, l}$ the constants defined in (28a). Then $\sum_{n} \sum_{l \leq n+2} c_{n, l}<\infty$. Now we apply (28a) to get

$$
\begin{aligned}
\left\|U_{1}\right\|_{1} & \left.\leq \sum_{\substack { l \leq n+2 \\
\begin{subarray}{c}{n+I \\
I \in \mathcal{I}^{\mu}{ l \leq n + 2 \\
\begin{subarray} { c } { n + I \\
I \in \mathcal { I } ^ { \mu } } }\end{subarray}} c_{n, l} \sum_{\substack{k, I \\
k:-k \leq L(I)}} \mid b_{k}^{\mu, I} \|_{2}^{2}\right)^{1 / 2} \\
& \lesssim \sum_{\substack{\mu, I: \\
I \in \mathcal{I}^{\mu}}}|I|^{1 / 2}\left(\sum_{k:-k \leq L(I)}\left\|b_{k}^{\mu, I}\right\|_{2}^{2}\right)^{1 / 2} .
\end{aligned}
$$

We apply a similar argument to estimate the $L^{1}$ norms of $U_{2}, U_{3}, U_{4}$. For $U_{2}$ we get

$$
\left\|U_{2}\right\|_{1} \leq \sum_{\substack{n \leq 2 \\ \ell \leq n-2}} \sum_{\substack{\mu, I: \\ I \in \mathcal{I}^{\mu}}}|I|^{1 / 2}\left(\iint\left\|\sum_{\substack{k:(s, t) \in \mathcal{W}_{k}^{n, l} \\-k \leq L(I)}} P_{k} T_{k} b_{k}^{\mu, I}(\cdot, s, t)\right\|_{2}^{2} \mathrm{~d} s \mathrm{~d} t\right)^{1 / 2}
$$




$$
\lesssim \sum_{\substack{\mu, I: \\ I \in \mathcal{I}^{\mu}}}|I|^{1 / 2}\left(\sum_{k:-k \leq L(I)}\left\|b_{k}^{\mu, I}\right\|_{2}^{2}\right)^{1 / 2}
$$

where we used (28d). By (28b)

$$
\begin{aligned}
\left\|U_{3}\right\|_{1} & \leq \sum_{\substack{\mu, I: \\
I \in \mathcal{I}^{\mu}}}|I|^{1 / 2} \sum_{\substack{n \geq 2 \\
\ell \leq n-2}}\left(\iint \sum_{\substack{k:(s, t) \in \mathcal{W}_{k}^{n, l} \\
-k+n \leq L(I)}} P_{k} T_{k, 1} b_{k}^{\mu, I}(\cdot, s, t) \|_{2}^{2} \mathrm{~d} s \mathrm{~d} t\right)^{1 / 2} \\
& \lesssim \sum_{\substack{\mu, I: \\
I \in \mathcal{I}^{\mu}}}|I|^{1 / 2}\left(\sum_{k:-k \leq L(I)}\left\|b_{k}^{\mu, I}\right\|_{2}^{2}\right)^{1 / 2},
\end{aligned}
$$

and, by (28c),

$$
\begin{aligned}
\left\|U_{4}\right\|_{1} & \leq \sum_{\substack{\mu, I: \\
I \in \mathcal{I}^{\mu}}}|I|^{1 / 2} \sum_{\substack{n \geq 2 \\
\ell \leq n-2}}\left(\iint \sum_{\substack{k:(s, t) \in \mathcal{W}_{k}^{n, l} \\
-k+l \leq L(I)}} P_{k} T_{k, 2} b_{k}^{\mu, I}(\cdot, s, t) \|_{2}^{2} \mathrm{~d} s \mathrm{~d} t\right)^{1 / 2} \\
& \lesssim \sum_{\substack{\mu, I: \\
I \in \mathcal{I}^{\mu}}}|I|^{1 / 2}\left(\sum_{k:-k \leq L(I)}\left\|b_{k}^{\mu, I}\right\|_{2}^{2}\right)^{1 / 2} .
\end{aligned}
$$

Finally we use $\sum_{k}\left\|b_{k}^{\mu, I}\right\|_{2}^{2}=\left(\gamma_{\mu}^{I}\right)^{2}$ in all estimates above to complete the proof of (47).

\section{Proof of (48)}

We have, by Minkowski integral inequality,

$$
\begin{aligned}
& V_{*}(x) \leq \sum_{\substack{n, l \\
n \geq \max \{0, l-2\}}}\left(\iint_{\mathcal{V}_{k}^{n, l}}\left|\sum_{k:(s, t) \in \mathcal{V}_{k}^{n, l}} \sum_{\substack{\mu, I: \\
I \in \mathcal{I}^{\mu} \\
\kappa(\mu, I)<-k+n}} P_{k} T_{k} b_{k}^{\mu, I}(x, s, t)\right|^{2} \mathrm{~d} s \mathrm{~d} t\right)^{1 / 2} \\
& \leq \sum_{\substack{n, l \\
n \geq \max \{0, l-2\}}}\left(\sum_{k} \iint_{\mathcal{V}_{k}^{n, l}}\left|\sum_{\substack{\mu, I: \\
I \in \mathcal{I}^{\mu} \\
\kappa(\mu, I)<-k+n}} P_{k} T_{k} b_{k}^{\mu, I}(x, s, t)\right|^{2} \mathrm{~d} s \mathrm{~d} t\right)^{1 / 2}
\end{aligned}
$$

and so, by Fubini,

$$
\left\|V_{*}\right\|_{2} \lesssim \sum_{\substack{n, l: \\ n \geq \max \{0, l-2\}}}\left(\sum_{k} \iint_{\mathcal{V}_{k}^{n, l}}\left\|\sum_{\substack{\mu, I: I \in \mathcal{I}^{\mu} \\ \kappa(\mu, I)<-k+n}} P_{k} T_{k} b_{k}^{\mu, I}(\cdot, s, t)\right\|_{2}^{2} \mathrm{~d} s \mathrm{~d} t\right)^{1 / 2} .
$$


By (28a),

$$
\begin{aligned}
\left\|V_{*}\right\|_{2} \lesssim & \sum_{l} \min \left\{2^{-l(\alpha-1 / 2)}, 2^{l(3 / 2-\alpha)}\right\} \sum_{n \geq \max \{0, l-3\}} 2^{-n / 2} \\
& \times\left(\sum_{k}\left\|\sum_{\substack{\mu, I: I \in \mathcal{I}^{\mu} \\
\kappa(\mu, I)<-k+n}} b_{k}^{\mu, I}\right\|_{2}^{1 / 2}\right)^{2} .
\end{aligned}
$$

For fixed $k$,

$$
\begin{aligned}
& \left\|\sum_{\mu} \sum_{\substack{I \in \mathcal{I}^{\mu}: \\
\kappa(\mu, I)<-k+n}} b_{k}^{\mu, I}\right\|_{2}^{2}=\left\|\sum_{\mu} \sum_{\substack{\mu, I: \\
\kappa(\mu, I)<-k+n}} \sum_{\substack{J \in \mathfrak{J}_{k}^{\mu} \\
I(J)=I}} \mathbb{1}_{J} L_{k} f\right\|_{2}^{2} \\
& =\sum_{\mu} \sum_{\substack{I \in \mathcal{I}^{\mu} \\
\kappa(\mu, I)<-k+n}} \sum_{\substack{J \in \mathcal{J}_{k}^{\mu} \\
I(J)=I}}\left\|\mathbb{1}_{J} L_{k} f\right\|_{2}^{2}
\end{aligned}
$$

because each dyadic interval of length $2^{-k}$ is contained in exactly one family $\mathfrak{J}_{k}^{\mu}$, and for fixed $\mu$ the intervals in $\mathcal{I}^{\mu}$ have disjoint interior. Now, since $1 / 2<\alpha<3 / 2$, we can sum in $l$ and obtain

$$
\begin{aligned}
\left\|V_{*}\right\|_{2}^{2} & \lesssim\left(\sum_{n \geq 0} 2^{-n}\left(\sum_{k} \sum_{\substack{\mu, I: I \in \mathcal{I}^{\mu} \\
\kappa(\mu, I)<-k+n}} \sum_{\substack{J \in \mathfrak{J}_{k}^{\mu} \\
I(J)=I}}\left\|\mathbb{1}_{J} L_{k} f\right\|_{2}^{2}\right)^{1 / 2}\right) \\
& \lesssim \sum_{k} \sum_{\substack{\mu, I: \\
I \in \mathcal{I}^{\mu}}} 2^{-(\kappa(\mu, I)+k)} \sum_{\substack{J \in \mathfrak{J}_{k}^{\mu} \\
I(J)=I}}\left\|\mathbb{1}_{J} L_{k} f\right\|_{2}^{2} \\
& \lesssim \sum_{\substack{\mu, I: \\
I \in \mathcal{I}^{\mu}}} 2^{-(\kappa(\mu, I)-L(I))} \sum_{\substack{J \in \mathfrak{J}_{k}^{\mu} \\
I(J)=I}} 2^{-(L(I)+k)}\left\|\mathbb{1}_{J} L_{k} f\right\|_{2}^{2}
\end{aligned}
$$

and hence

$$
\begin{aligned}
\left\|V_{*}\right\|_{2}^{2} & \lesssim \sum_{\mu, I: I \in \mathcal{I}^{\mu}} 2^{L(I)-\kappa(\mu, I)} \gamma_{\mu, I}^{2} \\
& \lesssim \sum_{\mu}\left[\sum_{\substack{I \in \mathcal{I}^{\mu} \\
\kappa(\mu, I)=L(I)}} \gamma_{\mu, I}^{2}+\sum_{\substack{I \in \mathcal{I}^{\mu} \\
\kappa(\mu, I)>L(I)}} 2^{L(I)-\kappa(\mu, I)} \gamma_{\mu, I}^{2}\right] .
\end{aligned}
$$

If $\kappa(\mu, I)=L(I)$ then $\tilde{\varkappa}(\mu, I) \leq L(I)$ and by definition of $\tilde{\varkappa}(\mu, I)$ we then have $2^{L(I)} \geq$ $2^{\tilde{\varkappa}(\mu, I)} \geq \lambda^{-1}|I|^{1 / 2} \gamma_{\mu, I}$. Thus $\gamma_{\mu, I} \leq|I|^{1 / 2} \lambda$. Therefore,

$$
\sum_{\substack{I \in \mathcal{I}^{\mu} \\ \kappa(\mu, I)=\dot{L}(I)}} \gamma_{\mu, I}^{2} \leq \lambda \sum_{I \in \mathcal{I}^{\mu}}|I|^{1 / 2} \gamma_{\mu, I} .
$$


If $\kappa(\mu, I)>L(I)$ then $\kappa(\mu, I)=\tilde{\varkappa}(\mu, I)$ and thus $2^{-\kappa(\mu, I)} \leq \lambda|I|^{-1 / 2} \gamma_{\mu, I}^{-1}$, again by the definition of $\tilde{\varkappa}(\mu, I)$. Hence,

$$
\sum_{\substack{I \in \mathcal{I}^{\mu} \\ \kappa(\mu, I)>L(I)}} 2^{L(I)-\kappa(\mu, I)} \gamma_{\mu, I}^{2} \lesssim \lambda \sum_{I \in \mathcal{I}^{\mu}}|I|^{1 / 2} \gamma_{\mu, I} .
$$

Now combining (51) with (52a), (52b) completes the proof of (48).

\section{Proof of (49)}

This proof follows the lines of that of (48). Notice that the conditions $\ell \geq \kappa(\mu, I)+k \geq$ $L(I)+k$ imply that $\ell \geq 0$. Now

$$
W_{2, *}(x) \leq \sum_{\substack{n, \ell \\ n \geq \max \{0, \ell+2\}}}\left(\sum_{k} \iint_{\mathcal{W}_{k}^{n, l}}\left|\sum_{\substack{\mu, I: I \in \mathcal{I}^{\mu} \\ \kappa(\mu, I)<-k+\ell}} P_{k} T_{k, 2} b_{k}^{\mu, I}(x, s, t)\right|^{2} \mathrm{~d} s \mathrm{~d} t\right)^{1 / 2}
$$

and so

$$
\left\|W_{2, *}\right\|_{2} \leq \sum_{\substack{n, \ell \\ n \geq \max \{0, \ell+2\}}}\left(\sum_{k} \iint_{\mathcal{W}_{k}^{n, l}}\left\|\sum_{\substack{\mu, I: I \in \mathcal{I}^{\mu} \\ \kappa(\mu, I)<-k+\ell}} P_{k} T_{k, 2} b_{k}^{\mu, I}(\cdot, s, t)\right\|_{2}^{2} \mathrm{~d} s \mathrm{~d} t\right)^{1 / 2} .
$$

By (28c)

$$
\begin{aligned}
\left\|W_{2, *}\right\|_{2}^{2} & \lesssim \sum_{n \geq 0} 2^{-n(2 \alpha-1)} \sum_{\ell \geq 0} 2^{-\ell} \sum_{k} \sum_{\substack{\mu, I: I \in \mathcal{I}^{\mu} \\
\kappa(\mu, I)<-k+\ell}} b_{k}^{\mu, I} \|_{2}^{2} \\
& \lesssim \sum_{\ell \geq 0} 2^{-\ell} \sum_{k} \sum_{\substack{\mu, I: I \in \mathcal{I}^{\mu} \\
\kappa(\mu, I)<-k+\ell}} \sum_{\substack{J \in \mathfrak{J}_{k}^{\mu} \\
I(J)=I}}\left\|\mathbb{1}_{J} L_{k} f\right\|_{2}^{2},
\end{aligned}
$$

which implies

$$
\left\|W_{2, *}\right\|_{2}^{2} \lesssim \sum_{k} \sum_{\substack{\mu, I: \\ I \in \mathcal{I}^{\mu}}} 2^{-k-\kappa(\mu, I)} \sum_{\substack{J \in \mathfrak{J}_{\mu}^{k} \\ I(J)=I}}\left\|\mathbb{1}_{J} L_{k} f\right\|_{2}^{2} \lesssim \sum_{\substack{\mu, I: \\ I \in \mathcal{I}^{\mu}}} 2^{L(I)-\kappa(\mu, I)} \gamma_{\mu, I}^{2}
$$

and this expression has been already estimated by $C \lambda \sum_{\mu, I}|I|^{1 / 2} \gamma_{\mu, I}$, by (51), (52a) and (52b). This finishes the proof of (49).

\section{Proof of (50)}

We now take advantage of the fact that the $L^{2}$ bounds for $T_{k, 1}$ in (28b) are somewhat better then the corresponding bounds for $T_{k, 2}$ in (28c). This allows us to invoke a straightforward $L^{1}$ estimate for $W_{1}$ as opposed to the $L^{2}$ arguments used for $V_{*}$ and $W_{2, *}$. We have 


$$
\begin{aligned}
\left\|W_{1}\right\|_{1} & \left.=\| \iint\left|\sum_{\substack{n, \ell: \\
n \geq \max \{0, \ell+2\}}} \sum_{\substack { k:(s, t) \in \mathcal{W}_{k}^{n, \ell} \\
\begin{subarray}{c}{\mu, I: I \in \mathcal{I}^{\mu} \\
L(I)<-k+n{ k : ( s , t ) \in \mathcal { W } _ { k } ^ { n , \ell } \\
\begin{subarray} { c } { \mu , I : I \in \mathcal { I } ^ { \mu } \\
L ( I ) < - k + n } }\end{subarray}} P_{k} T_{k, 1} b_{k}^{\mu, I}(\cdot, s, t)\right|^{2} \mathrm{~d} s \mathrm{~d} t\right)^{1 / 2} \|_{1} \\
& \lesssim \sum_{\substack{n, \ell: \\
n \geq \max \{0, \ell+2\}}} \sum_{\substack { \mu, I: \mathcal{I}^{\mu} \\
\begin{subarray}{c}{\mu \\
l \in L(I)-n<-k \leq L(I){ \mu , I : \mathcal { I } ^ { \mu } \\
\begin{subarray} { c } { \mu \\
l \in L ( I ) - n < - k \leq L ( I ) } }\end{subarray}}\left\|\left(\iint_{\mathcal{W}_{k}^{n, \ell}}\left|P_{k} T_{k, 1} b_{k}^{\mu, I}(\cdot, s, t)\right|^{2} \mathrm{~d} s \mathrm{~d} t\right)^{1 / 2}\right\|_{1} .
\end{aligned}
$$

Now observe that the expression inside $\|\cdots\|_{1}$ is supported in an interval of length $2^{-k+n+5}$, concentric with $I$. Hence, by the Cauchy-Schwarz inequality and Fubini

$$
\left\|W_{1}\right\|_{1} \lesssim \sum_{\substack{n, \ell: \\ n \geq \max \{0, \ell+2\}}} \sum_{\substack{\mu, I: \\ I \in \mathcal{I}^{\mu}}} \sum_{\substack{L(I)-n<\\-k \leq L(I)}} 2^{\frac{n-k}{2}}\left(\iint_{\mathcal{W}_{k}^{n, \ell}}\left\|P_{k} T_{k, 1} b_{k}^{\mu, I}(\cdot, s, t)\right\|_{2}^{2} \mathrm{~d} s \mathrm{~d} t\right)^{1 / 2} .
$$

By (28b),

$$
\begin{aligned}
\left\|W_{1}\right\|_{1} & \lesssim \sum_{\substack{n, \ell: \\
n \geq \max \{0, \ell+2\}}} 2^{\ell / 2} 2^{-n\left(\alpha+\frac{1}{2}\right)} \sum_{\substack{\mu, I: \\
I \in \mathcal{I}^{\mu}-k \leq L(I)}} \sum_{\substack{L(I)-n<\\
-k \leq L}} 2^{\frac{n-k}{2}}\left\|b_{k}^{\mu, I}\right\|_{2} \\
& \lesssim \sum_{n \geq 0} 2^{-n(\alpha-1 / 2)} \sum_{\substack{\mu, I: \\
I \in \mathcal{I}^{\mu}}} \sum_{\substack{n \leq L(I)\\
}} 2^{-(k+L(I)) / 2}|I|^{1 / 2}\left\|b_{k}^{\mu, I}\right\|_{2}
\end{aligned}
$$

and it follows easily that

$$
\left\|W_{1}\right\|_{1} \lesssim \sum_{\mu} \sum_{I \in \mathcal{I}^{\mu}}|I|^{1 / 2} \gamma_{\mu, I},
$$

as claimed.

\section{$8 L^{p}$ estimates}

\subsection{Proof of Theorem 1.1, via estimates on Hardy spaces.}

The lower bounds have already been established in Sect. 5. For the upper bounds we need to distinguish the case $1<p<2$ (for which the result is an immediate consequence of what we have already proved) and the case $2<p<\infty$.

\subsubsection{The case $1<p<2$}

For the upper $L^{p}$ bounds we note that

$$
\left\|S_{\alpha}\left(\mathcal{D}^{-\alpha} f\right)\right\|_{L^{p}} \leq C_{p}\|f\|_{L^{p}}, \quad 1<p<2,
$$

follows by real interpolation ([8]) from the already proved bounds

$$
\begin{aligned}
& \left\|S_{\alpha}\left(\mathcal{D}^{-\alpha} f\right)\right\|_{L^{1, \infty}} \lesssim\|f\|_{H^{1}}, \\
& \left\|S_{\alpha}\left(\mathcal{D}^{-\alpha} f\right)\right\|_{L^{2}} \lesssim\|f\|_{L^{2}} .
\end{aligned}
$$


8.1.2 The case $2<p<\infty$

Consider the operator $\mathcal{T}$ acting on the $\mathcal{H}=L^{2}(\mathbb{R} \times \mathbb{R})$ valued functions $F$ by

$$
\mathcal{T} F(x)=\iint_{|t| \leq|s|} \frac{s^{-1} \Delta_{-s} \mathcal{D}^{-\alpha} F(x, s, t)-t^{-1} \Delta_{-t} \mathcal{D}^{-\alpha} F(x, s, t)}{|s-t|^{\alpha}} \mathrm{d} s \mathrm{~d} t .
$$

and observe that (53) for $2 \leq p<\infty$ follows by duality from

$$
\|\mathcal{T} F\|_{L^{p}} \leq C_{p}\|F\|_{L^{p}(\mathcal{H})}, \quad 1<p \leq 2 .
$$

This can be deduced by real interpolation from

$$
\|\mathcal{T} F\|_{L^{2}} \lesssim\|F\|_{L^{2}(\mathcal{H})}
$$

(which is equivalent to the case $p=2$ of (53)) and

\section{Theorem 8.1}

$$
\|\mathcal{T} F\|_{L^{1, \infty}} \lesssim\|F\|_{H^{1}(\mathcal{H})}
$$

This result follows from

$$
\begin{aligned}
& \operatorname{meas}(\{x:|\mathcal{T} F(x)|>\lambda\}) \\
& \leq \frac{C}{\lambda}\left\|\left(\sum_{k \in \mathbb{Z}} \sup _{|h| \leq 2^{-k}} \iint\left|L_{k} F(\cdot+h, s, t)\right|^{2} \mathrm{~d} s \mathrm{~d} t\right)^{1 / 2}\right\|_{1}
\end{aligned}
$$

where the $L^{1}$ norm on the right hand side involves a version of the maximal square function $\mathfrak{S} F$ in (32), but for $\mathcal{H}$-valued functions $F$. More precisely, in (31) one should replace the absolute value by the norm in $\mathcal{H}$. Then, Peetre's estimate (33) holds in this context. The proof of (57) will be omitted since it is essentially the same as the proof of Theorem 1.2, with appropriate notational modifications.

\subsection{An alternative approach to Theorem 1.1}

There is an alternative (more straightforward and direct, but not less lengthy) approach to Theorem 1.1 which bypasses Theorem 1.2.

To be specific we let $\phi$ be a $C^{\infty}$ function supported in $\{\xi: 1 / 2<|\xi| \leq 2\}$ and let $\Phi=\mathcal{F}^{-1}[\phi]$. Let $K_{k}(x, s, t)$ be defined by

$$
\widehat{K}_{k}(\xi, s, t)=2^{-k \alpha}|s-t|^{-\alpha} \phi\left(2^{-k}|\xi|\right)\left(\frac{e^{i s \xi}-1}{s}-\frac{e^{i t \xi}-1}{t}\right) .
$$

By Littlewood-Paley theory one reduces the proof of Theorem 1.1 to the following $L^{p}$ inequalities for $1<p<2$ :

$$
\left\|\left(\sum_{k \in \mathbb{Z}} \iint_{|t| \leq|s|}\left|\int K_{k}(\cdot-y, s, t) f_{k}(y) \mathrm{d} y\right|^{2} \mathrm{~d} s \mathrm{~d} t\right)^{1 / 2}\right\|_{p} \leq C_{p}\left\|\left(\sum_{k}\left|f_{k}\right|^{2}\right)^{1 / 2}\right\|_{p},
$$


and

$$
\begin{aligned}
& \left\|\left(\sum_{k \in \mathbb{Z}}\left|\iint_{|t| \leq|s|} \int K_{k}(\cdot-y, s, t) F_{k}(y, s, t) \mathrm{d} y \mathrm{~d} s \mathrm{~d} t\right|^{2}\right)^{1 / 2}\right\|_{p} \\
& \leq C_{p}\left\|\left(\sum_{k} \iint\left|F_{k}(\cdot, s, t)\right|^{2} \mathrm{~d} s \mathrm{~d} t\right)^{1 / 2}\right\|_{p} .
\end{aligned}
$$

One decomposes, for each $k$, the half plane $\{|t| \leq|s|\}$ as a union of $\mathcal{V}_{k}^{n, l}$ and $\mathcal{W}_{k}^{n, \ell}$, as in Sect. 6. One then aims to prove, for $1<p \leq 2$, that there is $\varepsilon(p)>0$, such that

$$
\begin{aligned}
& \left\|\left(\sum_{k \in \mathbb{Z}} \iint_{\mathcal{V}_{k}^{n, l}}\left|\int K_{k}(\cdot-y, s, t) f_{k}(y) \mathrm{d} y\right|^{2} \mathrm{~d} s \mathrm{~d} t\right)^{1 / 2}\right\|_{p} \\
& \leq C_{p} 2^{-(|n|+|l|) \varepsilon(p)}\left\|\left(\sum_{k}\left|f_{k}\right|^{2}\right)^{1 / 2}\right\|_{p}, \\
& \left\|\left(\sum_{k \in \mathbb{Z}} \iint_{\mathcal{W}_{k}^{n, \ell}}\left|\int K_{k}(\cdot-y, s, t) f_{k}(y) \mathrm{d} y\right|^{2} \mathrm{~d} s \mathrm{~d} t\right)^{1 / 2}\right\|_{p} \\
& \leq C_{p} 2^{-(|n|+|l|) \varepsilon(p)}\left\|\left(\sum_{k}\left|f_{k}\right|^{2}\right)^{1 / 2}\right\|_{p},
\end{aligned}
$$

and also the dual versions (with $\mathcal{H}=L^{2}(\mathbb{R} \times \mathbb{R})$ )

$$
\begin{aligned}
& \left\|\left(\sum_{k \in \mathbb{Z}}\left|\iint_{\mathcal{V}_{k}^{n, l}} \int K_{k}(\cdot-y, s, t) F_{k}(y, s, t) \mathrm{d} y \mathrm{~d} s \mathrm{~d} t\right|^{2}\right)^{1 / 2}\right\|_{p} \\
& \leq C_{p} 2^{-(|n|+|l|) \varepsilon(p)}\left\|\left(\sum_{k}\left|F_{k}\right|_{\mathcal{H}}^{2}\right)^{1 / 2}\right\|_{p}, \\
& \left\|\left(\sum_{k \in \mathbb{Z}}\left|\iint_{\mathcal{W}_{k}^{n, \ell}} \int K_{k}(\cdot-y, s, t) F_{k}(y, s, t) \mathrm{d} y \mathrm{~d} s \mathrm{~d} t\right|^{2}\right)^{1 / 2}\right\|_{p} \\
& \quad \leq C_{p} 2^{-(|n|+|\ell|) \varepsilon(p)}\left\|\left(\sum_{k}\left|F_{k}\right|_{\mathcal{H}}^{2}\right)^{1 / 2}\right\|_{p} .
\end{aligned}
$$

For $p=2$ such estimates follow from Sect. 6. For $p=1$ one proves slightly weaker $L^{1} \rightarrow L^{1, \infty}$ inequalities, with constants $O(1+|n|+|l|)$ and $O(1+|n|+|\ell|)$, respectively. These follow if one checks the Hörmander condition on the kernel $K_{k}, c f$. [9] and [17], namely 


$$
\begin{aligned}
& \int_{|x| \geq 2 h}\left(\sum_{k \in \mathbb{Z}} \iint_{\mathcal{V}_{k}^{n, l}}\left|K_{k}(x+h, s, t)-K_{k}(x, s, t)\right|^{2} \mathrm{~d} s \mathrm{~d} t\right)^{1 / 2} \mathrm{~d} x \\
& \quad \lesssim 1+|n|+|l|
\end{aligned}
$$

and

$$
\begin{aligned}
& \int_{|x| \geq 2 h}\left(\sum_{k \in \mathbb{Z}} \iint_{\mathcal{W}_{k}^{n, \ell}}\left|K_{k}(x+h, s, t)-K_{k}(x, s, t)\right|^{2} \mathrm{~d} s \mathrm{~d} t\right)^{1 / 2} \mathrm{~d} x \\
& \quad \lesssim 1+|n|+|\ell| .
\end{aligned}
$$

In fact slightly better bounds than (60a), (60b) can be proved, but they are not good enough to sum in all the parameters $(n, l),(n, \ell)$, respectively. Inequalities $(60 \mathrm{a}),(60 \mathrm{~b})$ can be established by straightforward $L^{1}$ and $L^{2}$ estimates used earlier; we shall not include the details. One can interpolate the weak type $(1,1)$ inequalities implied by $(60 \mathrm{a}),(60 \mathrm{~b})$ and the improved $L^{2}$ results to show the $L^{p}$ inequalities (58) and (59), and these yield a proof of Theorem 1.1.

\section{Pointwise differentiability}

Let $f \in L^{2}(\mathbb{R})$. A classical result of Stein and Zygmund [19], [17, ch. VIII] says that $f$ is differentiable at almost every point $x \in \mathbb{R}$ for which there exists $\delta=\delta(x)>0$ such that

$$
\sup _{|t|<\delta}\left|\frac{f(x+2 t)-f(x)}{2 t}-\frac{f(x+t)-f(x)}{t}\right|<\infty
$$

and

$$
\int_{|t|<\delta}\left|\frac{f(x+2 t)-f(x)}{2 t}-\frac{f(x+t)-f(x)}{t}\right|^{2} \frac{\mathrm{d} t}{|t|}<\infty .
$$

Conversely, for almost every point $x \in \mathbb{R}$ where $f$ is differentiable there exists $\delta=\delta(x)>0$ such that (62) holds. Notice that (61) is the Zygmund condition at $x$ in disguise.

The purpose of this section is to discuss analogous results when the integral in (62) is replaced by local versions of $S_{\alpha} f$ for $\alpha=1$, the square function of the previous sections. We drop the subscript and write $S f \equiv S_{1} f$.

\subsection{Preliminary considerations}

\subsubsection{Marcinkiewicz integrals}

The following classical result on Marcinkiewicz integrals is a crucial tool in proving results on pointwise differentiation.

Let $F$ be a closed set of positive measure, and fix $\lambda>0$. Let

$$
I^{(\lambda)}(x):=\int_{x-1}^{x+1} \frac{\operatorname{dist}^{\lambda}(y, F)}{|x-y|^{1+\lambda}} \mathrm{d} y .
$$

Then, one proves $[17, \mathrm{p} .15]$ that

$$
I^{(\lambda)}(x)<\infty \text { for almost every } x \in F .
$$




\subsubsection{Pointwise comparison with a related square function}

Given $f \in L^{2}(\mathbb{R})$ and $m \in \mathbb{R}$, consider the square functions $Q f$ defined for $x \in \mathbb{R}$ by

$$
Q f(x)=\left(\int_{1<|m| \leq 2} \int_{\mathbb{R}}\left|\frac{f(x+m t)-f(x+t)}{(m-1) t}-\frac{f(x+t)-f(x)}{t}\right|^{2} \frac{\mathrm{d} t}{|t|} \mathrm{d} m\right)^{1 / 2} .
$$

We shall use the identity

$$
\begin{aligned}
& \frac{f(x+m t)-f(x)}{m t}-\frac{f(x+t)-f(x)}{t} \\
& \quad=\frac{m-1}{m}\left(\frac{f(x+m t)-f(x+t)}{(m-1) t}-\frac{f(x+t)-f(x)}{t}\right)
\end{aligned}
$$

to show that $Q f$ and $S f$ are equivalent.

Lemma 9.1 There exists a constant $C>0$ such that

$$
C^{-1} Q f(x) \leq S f(x) \leq C Q f(x), \quad x \in \mathbb{R}, \quad f \in L^{2}(\mathbb{R}) .
$$

Proof Fix $u \in \mathbb{R}$ and $N \geq 1$. Since

$$
\begin{aligned}
& \frac{f\left(x+u^{N} t\right)-f(x)}{u^{N} t}-\frac{f(x+t)-f(x)}{t} \\
& =\sum_{j=1}^{N} \frac{f\left(x+u^{j} t\right)-f(x)}{u^{j} t}-\frac{f\left(x+u^{j-1} t\right)-f(x)}{u^{j-1} t},
\end{aligned}
$$

Schwarz's inequality gives

$$
\begin{aligned}
& \left|\frac{f\left(x+u^{N} t\right)-f(x)}{u^{N} t}-\frac{f(x+t)-f(x)}{t}\right|^{2} \\
& \quad \leq N \sum_{j=1}^{N}\left|\frac{f\left(x+u^{j} t\right)-f(x)}{u^{j} t}-\frac{f\left(x+u^{j-1} t\right)-f(x)}{u^{j-1} t}\right|^{2} .
\end{aligned}
$$

Hence, with $\mathcal{G}_{1, m}$ as in (9),

$$
\begin{aligned}
\mathcal{G}_{1, u^{N}} f(x)^{2} & \leq N \sum_{j=1}^{N} \int_{\mathbb{R}}\left|\frac{f\left(x+u^{j} t\right)-f(x)}{u^{j} t}-\frac{f\left(x+u^{j-1} t\right)-f(x)}{u^{j-1} t}\right|^{2} \frac{\mathrm{d} t}{|t|} \\
& =N^{2} \mathcal{G}_{1, u} f(x)^{2} .
\end{aligned}
$$

Fix $0<\varepsilon<1$. We perform the change of variable $m=u^{N}$ and then estimate

$$
\begin{aligned}
& \int_{(1+\varepsilon)^{N}}^{2^{N}} \mathcal{G}_{1, m} f(x)^{2} \frac{\mathrm{d} m}{(m-1)^{2}}=\int_{1+\varepsilon}^{2} \mathcal{G}_{1, u^{N}} f(x)^{2} \frac{N u^{N-1}}{\left(u^{N}-1\right)^{2}} \mathrm{~d} u \\
& \leq N \int_{1+\varepsilon}^{2} \mathcal{G}_{1, u^{N}} f(x)^{2} \frac{1}{u^{N-1}} \frac{\mathrm{d} u}{(u-1)^{2}} \leq \frac{N^{3}}{(1+\varepsilon)^{N-1}} \int_{1+\varepsilon}^{2} \mathcal{G}_{1, u} f(x)^{2} \frac{\mathrm{d} u}{(u-1)^{2}} .
\end{aligned}
$$

A similar argument gives

$$
\int_{-2^{N}}^{-(1+\varepsilon)^{N}} \mathcal{G}_{1, m} f(x)^{2} \frac{\mathrm{d} m}{(m-1)^{2}} \leq \frac{N^{3}}{(1+\varepsilon)^{N-1}} \int_{-2}^{-1-\varepsilon} \mathcal{G}_{1, u} f(x)^{2} \frac{\mathrm{d} u}{(u-1)^{2}} .
$$


Thus

$$
\int_{|m| \geq 2} \mathcal{G}_{1, m} f(x)^{2} \frac{\mathrm{d} m}{(m-1)^{2}} \lesssim \int_{1<|m| \leq 2} \mathcal{G}_{1, m} f(x)^{2} \frac{\mathrm{d} m}{(m-1)^{2}} .
$$

By the identity (10) we get

$$
S f(x)^{2} \approx \int_{1<|m| \leq 2} \mathcal{G}_{1, m} f(x)^{2} \frac{\mathrm{d} m}{(m-1)^{2}}
$$

and the asserted equivalence follows immediately from the identity (64).

\subsubsection{An inequality for functions in the Zygmund class}

For the proof of Theorems 1.4 and 9.3 we need the following.

Lemma 9.2 Let $f \in \Lambda_{*}$. Then, there is a constant $C$ such that

$$
\begin{gathered}
\sup _{x \in \mathbb{R}|t| \leq 1}\left|\frac{f(x+m t)-f(x)}{m t}-\frac{f(x+t)-f(x)}{t}\right| \\
\leq C\|f\|_{\Lambda_{*}}|m-1|\left(1+\log \left(|m-1|^{-1}\right)\right),
\end{gathered}
$$

for $1<|m| \leq 2$.

Proof We shall use that divided differences of functions in $\Lambda^{*}$ satisfy a mild regularity property, namely

$$
\left|\frac{f(x+t)-f(x)}{t}-\frac{f(x+s)-f(x)}{s}\right| \leq C\|f\|_{\Lambda_{*}}
$$

for $x, t, s \in \mathbb{R}$ with $|s| / 2 \leq|t| \leq|s|$, see [6, Lemma 2]. This implies in particular the easier version of (73) where the sup is just taken over $m \in[-2,-1]$.

Now if $1<m \leq 2$ we apply the crucial identity (64) to gain the factor $m-1$; we then see that it suffices to show, for any $x, t \in \mathbb{R}$ and $1<m \leq 2$,

$$
\left|\frac{f(x+m t)-f(x+t)}{(m-1) t}-\frac{f(x+t)-f(x)}{t}\right| \lesssim\|f\|_{\Lambda_{*}}\left(1+\log \frac{1}{m-1}\right) .
$$

Let $N$ be the positive integer satisfying $1<2^{N}(m-1) \leq 2$. Since

$$
\left|\frac{\left.f\left(x+t+2^{k-1}(m-1) t\right)\right)-f(x+t)}{2^{k-1}(m-1) t}-\frac{f\left(x+t+2^{k}(m-1) t\right)-f(x+t)}{2^{k}(m-1) t}\right|
$$

is bounded by $C\|f\|_{\Lambda_{*}}$, uniformly in $x, t, k, m$, we obtain, summing in $k=1, \ldots, N$,

$$
\left|\frac{f(x+m t)-f(x+t)}{(m-1) t}-\frac{f\left(x+t+2^{N}(m-1) t\right)-f(x+t)}{2^{N}(m-1) t}\right| \lesssim N\|f\|_{\Lambda_{*}} .
$$

This gives (67). 


\subsection{Differentiability versus finiteness of a square function: an example}

We shall consider for any $\delta>0$ the local version $S_{\mathrm{loc}, \delta}$ of $S$, defined by

$$
S_{\mathrm{loc}, \delta} f(x)=\left(\iint_{|t|+|s|<\delta}\left|\frac{f(x+t)-f(x)}{t}-\frac{f(x+s)-f(x)}{s}\right|^{2} \frac{\mathrm{d} s \mathrm{~d} t}{|s-t|^{2}}\right)^{1 / 2} .
$$

We show that the finiteness of $S_{\mathrm{loc}, \delta} f(x)$ is generally not a necessary condition for differentiability. Specifically we present an example of a function $f$ differentiable at almost every point of a set $E$ of positive measure such that for any $\delta>0$,

$$
S_{\mathrm{loc}, \delta} f(x)=\infty \text {, for a.e. } x \in E \text {. }
$$

Hence, an analogue of the result of Stein and Zygmund in this context does not hold without additional assumptions on the function $f$ (such as for example the Zygmund class condition in Theorem 1.4).

Let $E \subset \mathbb{R}$ be a closed set of positive Lebesgue measure without interior points. Write $\mathbb{R} \backslash E=\cup I_{j}$, where $I_{j}=\left(c_{j}-b_{j}, c_{j}+b_{j}\right)$ are pairwise disjoint open intervals. We denote by $I_{j}^{\text {half }}=\left(c_{j}-b_{j} / 2, c_{j}+b_{j} / 2\right)$ the inner half of $I_{j}$. Let $f: \mathbb{R} \rightarrow \mathbb{R}$ satisfying

$$
|f(x)| \leq \operatorname{dist}(x, E), \quad x \in \mathbb{R}
$$

and, for each $j$,

$$
\int_{0}^{\delta}\left|\frac{f(y+u)-f(y)}{u}\right|^{2} \mathrm{~d} u=\infty \text { for all } y \in I_{j}^{\text {half }} .
$$

The change of variable $s=m t$ and identity (70) gives

$$
S_{\mathrm{loc}, \delta}^{2} f(x) \approx \int_{|t|<\delta} \int_{|m|>1}\left(\frac{f(x+m t)-f(x+t)}{(m-1) t}-\frac{f(x+t)-f(x)}{t}\right)^{2} \mathrm{~d} m \frac{\mathrm{d} t}{|t|} .
$$

We apply now Stepanov's theorem ([17, VIII, Thm.3] or [10]). It says that $f$ is differentiable at almost every point in $E$ if and only if $f\left(x_{0}+y\right)-f\left(x_{0}\right)=O(|y|)$ as $|y| \rightarrow 0$ for almost every $x_{0} \in E$. Hence, condition (69) implies that $f$ is differentiable at almost every point of $E$. Moreover, (69) and the Marcinkiewicz inequality (63) for $\lambda=2$ imply

$$
\int_{|t|<\delta}\left(\frac{f(x+t)}{t}\right)^{2} \frac{\mathrm{d} t}{|t|}<\infty, \text { for a.e. } x \in E .
$$

On the other hand, the change of variable $(m-1) t=u$ gives

$$
\begin{aligned}
& \int_{|t|<\delta} \int_{|m|>1}\left(\frac{f(x+m t)-f(x+t)}{(m-1) t}\right)^{2} \mathrm{~d} m \frac{\mathrm{d} t}{|t|} \\
& \geq \int_{|t|<\delta} \int_{0}^{1}\left(\frac{f(x+t+u)-f(x+t)}{u}\right)^{2} \mathrm{~d} u \frac{\mathrm{d} t}{|t|^{2}} .
\end{aligned}
$$

Now, for fixed $\delta>0$, for almost every $x \in E$ the interval $(x-\delta, x+\delta)$ contains an interval $I_{j}$. Here we use the assumption that $E$ is a closed set with no interior points. Hence, there exists a set of points $t \in(-\delta, \delta)$ of positive measure such that $x+t \in I_{j}^{\text {half }}$ and condition (70) shows that the last integral diverges. Hence, $S_{\mathrm{loc}, \delta} f(x)=\infty$ for almost every $x \in E$. 


\subsection{The main result on pointwise differentiability}

We shall now consider functions that are locally in the Zygmund class, i.e., satisfy condition (71) below. This condition clearly holds when $f$ is differentiable at $x$, but it is substantially weaker.

Theorem 9.3 Let $f \in L_{\text {loc }}^{2}(\mathbb{R})$.

a) The function $f$ is differentiable at almost every point $x \in \mathbb{R}$ where the following two conditions hold

$$
\limsup _{|h| \rightarrow 0}\left|\frac{f(x+2 h)-f(x)}{2 h}-\frac{f(x+h)-f(x)}{h}\right|<\infty
$$

and there exists $\delta=\delta(x)>0$ such that

$$
\iint_{|s|+|t|<\delta}\left|\frac{f(x+s)-f(x)}{s}-\frac{f(x+t)-f(x)}{t}\right|^{2} \frac{\mathrm{d} s \mathrm{~d} t}{|s-t|^{2}}<\infty .
$$

b) For almost every point $x \in \mathbb{R}$ where $f$ is differentiable and

$$
\limsup _{|t| \rightarrow 0} \sup _{1<|m| \leq 2} \frac{\left|\frac{f(x+m t)-f(x)}{m t}-\frac{f(x+t)-f(x)}{t}\right|}{|m-1|\left(1+\left|\log \frac{1}{|m-1|}\right|\right)}<\infty,
$$

there exists $\delta=\delta(x)>0$ such that

$$
\iint_{|s|+|t|<\delta}\left|\frac{f(x+s)-f(x)}{s}-\frac{f(x+t)-f(x)}{t}\right|^{2} \frac{\mathrm{d} s \mathrm{~d} t}{|s-t|^{2}}<\infty .
$$

Proof of Theorem 1.4 One direction is immediate by a) of Theorem 9.3. For the other direction one needs to verify that condition (73) holds for any $f \in \Lambda_{*}$. But this was proved in Lemma 9.2.

Proof of Theorem 9.3 (a) Given $\delta>0$, let $E=E^{\delta}$ be the set of points $x \in \mathbb{R}$ for which

$$
\iint_{|t|+|s|<\delta}\left|\frac{f(x+t)-f(x)}{t}-\frac{f(x+s)-f(x)}{s}\right|^{2} \frac{\mathrm{d} s \mathrm{~d} t}{|s-t|^{2}}<\delta^{-1}
$$

and

$$
\sup _{|h|<\delta}\left|\frac{f(x+2 h)-f(x)}{2 h}-\frac{f(x+h)-f(x)}{h}\right|<\delta^{-1} .
$$

We show that for any fixed $\delta>0$, the function $f$ is differentiable at almost every point of $E^{\delta}$. We can assume that $f$ vanishes outside an interval $I$ of length $\delta$. For $x \in E^{\delta} \cap I$ we have $S f(x)<\infty$ and Lemma 3.1 gives $G_{1} f(x)=2 \mathcal{G}_{1,2} f(x)<\infty$. Now, the Stein-Zygmund result gives that $f$ is differentiable at almost every point of $E^{\delta} \cap I$. The assertion a) follows if we consider the union $\cup_{j>1} E^{1 / j}$.

(b) Given $\delta>0$, let $E(\delta)$ be the set of points $x \in \mathbb{R}$ for which

$$
|f(x+t)-f(x)| \leq \delta^{-1}|t|,
$$

holds for $0 \leq|t| \leq \delta$ and

$$
\left|\frac{f(x+m t)-f(x)}{m t}-\frac{f(x+t)-f(x)}{t}\right| \leq \delta^{-1}|m-1|\left|1+\log \frac{1}{|m-1|}\right|
$$


holds when $1<|m| \leq 2$, and $0<|t| \leq \delta$. It suffices to show that condition (74) holds for almost every point $x \in E(\delta)$ for each given $\delta$ (then one takes the union $\cup_{j} E(1 / j)$ ) Without loss of generality we can assume that $E(\delta)$ is compact and that $f$ vanishes outside an interval $I$ of length $\delta$.

Given $\varepsilon>0$, we prove that the set of all $x \in E(\delta)$ where (74) fails is of measure less than $\varepsilon$. We can find a compact set $F \subset E(\delta)$ with $|E(\delta) \backslash F|<\varepsilon$ and a decomposition $f=g+b$ where $g$ is Lipschitz on $\mathbb{R}$ and $b$ vanishes on $F$ ([17, p. 248]). Moreover, we can also assume that $g$ and $b$ vanish outside $I^{*}$, the double interval with the same center. Applying the $L^{2}$ inequality for $\operatorname{Sg}$ we get $\operatorname{Sg}(x)<\infty$ for almost every $x \in \mathbb{R}$. Hence, we need to show that

$$
S b(x)<\infty \text { for almost every } x \in F \cap I .
$$

Since $g$ is Lipschitz on $\mathbb{R}$ we get

$$
\sup _{x \in \mathbb{R}} \sup _{|t| \leq \delta} \frac{|g(x+t)-g(x)|}{|t|} \leq C_{1},
$$

and since the Lipschitz space is contained in the Zygmund class we also have by Lemma 9.2

$$
\sup _{x \in \mathbb{R}} \sup _{|t| \leq \delta}\left|\frac{g(x+m t)-b(x)}{m t}-\frac{g(x+t)-b(x)}{t}\right| \leq C_{2}|m-1|\left(1+\log \frac{1}{|m-1|}\right)
$$

for $1 \leq|m| \leq 2$.

Therefore, the function $b$ satisfies, for some positive constant $A$,

$$
\sup _{|t| \leq \delta} \frac{|b(x+t)-b(x)|}{|t|} \leq A
$$

and

$$
\sup _{|t| \leq \delta}\left|\frac{b(x+m t)-b(x)}{m t}-\frac{b(x+t)-b(x)}{t}\right| \leq A|m-1|\left(1+\log \frac{1}{|m-1|}\right)
$$

for all $x \in F$ and $1 \leq|m| \leq 2$.

For the remainder of this proof implicit constants in inequalities of the form $\lesssim$ may depend on $A$.

Consider a Whitney decomposition of the open set $I^{*} \backslash \bar{I} \cap F$, that is, $I^{*} \backslash \bar{I} \cap F=\cup I_{j}$, where $\left\{I_{j}\right\}$ are pairwise disjoint intervals with

$$
\left|I_{j}\right| \leq \operatorname{dist}\left(I_{j}, \bar{I} \cap F\right) \leq 4\left|I_{j}\right| .
$$

Set $\bar{I}_{j}=\left[a_{j}, b_{j}\right]$ and let $x_{j}=\left(a_{j}+b_{j}\right) / 2$ denote the center of $I_{j}$. We let $I_{j}^{*}=\left(x_{j}-\right.$ $\left.\left|I_{j}\right|, x_{j}+\left|I_{j}\right|\right)$ denote the open double interval. By (63)

$$
\sum_{j} \frac{\left|I_{j}\right|^{1+\lambda}}{\left|x-x_{j}\right|^{1+\lambda}} \lesssim I^{\lambda}(x)<\infty
$$

for almost every $x \in I \cap F$. The plan of the proof is to show that there exists $\lambda>0$ such that

$$
|S b(x)|^{2} \lesssim \sum_{j} \frac{\left|I_{j}\right|^{1+\lambda}}{\left|x-x_{j}\right|^{1+\lambda}}
$$

for almost every $x \in F \cap I$.

By part (a) of Lemma 9.1 we have $S b(x) \approx Q b(x)$. Write

$$
L=\{m \in \mathbb{R}: 1<|m| \leq 2\} .
$$


Then, $|Q b(x)|^{2} \lesssim A(x)+B(x)$, where

$$
\begin{aligned}
& A(x)=\int_{\mathbb{R}}\left|\frac{b(x+t)-b(x)}{t}\right|^{2} \frac{\mathrm{d} t}{|t|}, \\
& B(x)=\int_{L} \int_{\mathbb{R}}\left|\frac{b(x+m t)-b(x+t)}{(m-1) t}\right|^{2} \frac{\mathrm{d} t}{|t|} \mathrm{d} m .
\end{aligned}
$$

We need to show that $A(x)<\infty, B(x)<\infty$ for almost every $x \in F \cap I$. In what follows we will always assume $x \in F \cap I$.

Since $b_{\mid F} \equiv 0$, condition (77) gives that $\sup _{x \in I_{j}}|b(x)| \lesssim\left|I_{j}\right|$ and hence

$$
\int_{I_{j}}|b|^{2} \lesssim\left|I_{j}\right|^{3}
$$

Therefore

$$
A(x)=\int_{\mathbb{R}} \frac{b(y)^{2}}{|y-x|^{3}} \mathrm{~d} y \lesssim \sum_{j} \frac{\left|I_{j}\right|^{3}}{\left|x_{j}-x\right|^{3}}
$$

which by (79) is finite for almost every $x \in F \cap I$.

Now $B(x)=C(x)+D(x)$ where

$$
\begin{aligned}
& C(x)=\int_{L} \int_{F-x}\left|\frac{b(x+m t)}{(m-1) t}\right|^{2} \frac{\mathrm{d} t}{|t|} \mathrm{d} m, \\
& D(x)=\int_{L} \int_{\mathbb{R} \backslash(F-x)}\left|\frac{b(x+m t)-b(x+t)}{(m-1) t}\right|^{2} \frac{\mathrm{d} t}{|t|} \mathrm{d} m .
\end{aligned}
$$

For each $m \in L$ and $j=1,2, \ldots c$, let $I_{j}(m)=\frac{I_{j}-x}{m}$. We have

$$
C(x)=\int_{L} \sum_{j} \int_{t \in(F-x) \cap I_{j}(m)}\left|\frac{b(x+m t)}{(m-1) t}\right|^{2} \frac{\mathrm{d} t}{|t|} \mathrm{d} m .
$$

Since $b_{\mid F} \equiv 0$, condition (77) gives that $\sup \left\{|b(x+m t)|: t \in I_{j}(m)\right\} \lesssim\left|I_{j}\right|$. Hence,

$$
C(x) \lesssim \int_{L} \sum_{j}\left|I_{j}\right|^{2}\left(\int_{t \in(F-x) \cap I_{j}(m)} \frac{\mathrm{d} t}{|t|^{3}}\right) \frac{\mathrm{d} m}{(m-1)^{2}}
$$

Now for each $t \in F-x$ we have $t \in I_{j}(m)$ if and only if $m \in I_{j}(t)$. Write $I_{j}=\left(a_{j}, b_{j}\right)$. Then, $I_{j}(t)=\left(\left(a_{j}-x\right) / t,\left(b_{j}-x\right) / t\right)$ and

$$
\int_{I_{j}(t)} \frac{\mathrm{d} m}{(m-1)^{2}} \leq\left|\frac{t}{a_{j}-x-t}-\frac{t}{b_{j}-x-t}\right|=|t| \frac{\left|I_{j}\right|}{\left|a_{j}-x-t\right|\left|b_{j}-x-t\right|} .
$$

Since $x+t \in F$ we have that both $\left|a_{j}-(x+t)\right|$ and $\left|b_{j}-(x+t)\right|$ are comparable to $\left|x_{j}-(x+t)\right|$. Then,

$$
\int_{I_{j}(t)} \frac{\mathrm{d} m}{(m-1)^{2}} \lesssim \frac{|t|\left|I_{j}\right|}{\left|x_{j}-(x+t)\right|^{2}}
$$


Since $x+t \in F$ we have $\left|x+t-x_{j}\right| \gtrsim\left|I_{j}\right|$. Moreover, from $m \in I_{j}(t),|m| \leq 2$ and $x \in F$ we have $|t| \geq\left|a_{j}-x\right| / 2 \approx\left|x-x_{j}\right|$. Fubini's theorem gives

$$
\begin{aligned}
C(x) & \lesssim \sum_{j}\left|I_{j}\right|^{3} \int_{\substack{|t| \gtrsim\left|x-x_{j}\right| \\
\left|x+t-x_{j}\right| \gtrsim\left|I_{j}\right|}} \frac{\mathrm{d} t}{\left|x_{j}-(x+t)\right|^{2}|t|^{2}} \\
& \lesssim \sum_{j} \frac{\left|I_{j}\right|^{3}}{\left|x-x_{j}\right|^{2}} \int_{\left|x+t-x_{j}\right| \gtrsim\left|I_{j}\right|} \frac{\mathrm{d} t}{\left|x_{j}-(x+t)\right|^{2}} \lesssim \sum_{j} \frac{\left|I_{j}\right|^{3}}{\left|x-x_{j}\right|^{3}},
\end{aligned}
$$

which by (79) is finite a.e. $x \in F$.

Next we estimate $D(x)$. By Fubini's theorem,

$$
D(x)=\sum_{j} \int_{a_{j}-x}^{b_{j}-x} \int_{L}\left|\frac{b(x+m t)-b(x+t)}{(m-1) t}\right|^{2} \mathrm{~d} m \frac{\mathrm{d} t}{|t|}=\sum_{j} E_{j}(x)+F_{j}(x),
$$

where

$$
\begin{aligned}
& E_{j}(x)=\int_{a_{j}-x}^{b_{j}-x} \int_{\{m \in L: x+m t \in F\}}\left|\frac{b(x+t)}{(m-1) t}\right|^{2} \mathrm{~d} m \frac{\mathrm{d} t}{|t|}, \\
& F_{j}(x)=\int_{a_{j}-x}^{b_{j}-x} \int_{\{m \in L: x+m t \notin F\}}\left|\frac{b(x+m t)-b(x+t)}{(m-1) t}\right|^{2} \mathrm{~d} m \frac{\mathrm{d} t}{|t|} .
\end{aligned}
$$

Note that since $\left\{I_{j}\right\}$ are Whitney intervals, $x+t \in I_{j}$ and $x+m t \in F$ implies that $|m-1| \gtrsim$ $\left|I_{j}\right| /\left|x-x_{j}\right|$. Hence,

$$
E_{j}(x) \lesssim \int_{a_{j}-x}^{b_{j}-x} \frac{|b(x+t)|^{2}}{|t|^{3}} \frac{\left|x-x_{j}\right|}{\left|I_{j}\right|} \mathrm{d} t .
$$

Since $b_{\mid F} \equiv 0$ condition (77) gives that $|b(x+t)| \lesssim\left|I_{j}\right|$ for $t \in\left(a_{j}-x, b_{j}-x\right)$. We have $|t| \approx\left|x-x_{j}\right|$ for any $t \in\left(a_{j}-x, b_{j}-x\right)$ because the $I_{j}$ are Whitney intervals.

It follows that

$$
E_{j}(x) \lesssim \frac{\left|I_{j}\right|^{2}}{\left|x-x_{j}\right|^{2}}
$$

and

$$
\sum_{j} E_{j}(x) \lesssim \sum_{j} \frac{\left|I_{j}\right|^{2}}{\left(x-x_{j}\right)^{2}}
$$

which by (79) is finite a.e. $x \in F$.

Let us now estimate $F_{j}(x)$. Split $F_{j}(x)=G_{j}(x)+H_{j}(x)$ where

$$
\begin{aligned}
& G_{j}(x)=\int_{a_{j}-x}^{b_{j}-x} \int_{\left\{m \in L: x+m t \in I_{j}^{*}\right\}}\left|\frac{b(x+m t)-b(x+t)}{(m-1) t}\right|^{2} \mathrm{~d} m \frac{\mathrm{d} t}{|t|}, \\
& H_{j}(x)=\int_{a_{j}-x}^{b_{j}-x} \sum_{k \neq j} \int_{\left\{m \in L: x+m t \in I_{k} \backslash I_{j}^{*}\right\}}\left|\frac{b(x+m t)-b(x+t)}{(m-1) t}\right|^{2} \mathrm{~d} m \frac{\mathrm{d} t}{|t|} .
\end{aligned}
$$


Recall that $I_{j}^{*}$ denotes the interval of double length centered at $x_{j}$. Now, the assumption (73) and identity (64) give that

$$
\left|\frac{b(x+m t)-b(x+t)}{(m-1) t}-\frac{b(x+t)-b(x)}{t}\right| \lesssim\left|\ln \frac{1}{|m-1|}\right| .
$$

Hence, $G_{j}(x)$ is bounded by a fixed multiple of

$$
\int_{a_{j}-x}^{b_{j}-x} \int_{\left\{m \in L: x+m t \in I_{j}^{*}\right\}} \ln ^{2} \frac{1}{|m-1|} \mathrm{d} m \frac{\mathrm{d} t}{|t|}+\int_{a_{j}-x}^{b_{j}-x}\left|\frac{b(x+t)-b(x)}{t}\right|^{2} \frac{\mathrm{d} t}{|t|} .
$$

Since $\left\{I_{j}\right\}$ are Whitney intervals, the fact that $x+t \in I_{j}$ and $x+m t \in I_{j}^{*}$ implies that $|m-1| \lesssim \frac{\left|I_{j}\right|}{\left|x-x_{j}\right|}$. So

$$
\int_{\left\{m \in L: x+m t \in I_{j}^{*}\right\}} \ln ^{2} \frac{1}{|m-1|} \mathrm{d} m \lesssim \frac{\left|I_{j}\right|}{\left|x-x_{j}\right|} \ln ^{2} \frac{\left|I_{j}\right|}{\left|x-x_{j}\right|}
$$

and we deduce that

$$
\int_{a_{j}-x}^{b_{j}-x} \int_{\left\{m \in L: x+m t \in I_{j}^{*}\right\}} \ln ^{2} \frac{1}{|m-1|} \mathrm{d} m \frac{\mathrm{d} t}{|t|} \lesssim \frac{\left|I_{j}\right|^{2}}{\left|x-x_{j}\right|^{2}} \ln ^{2} \frac{\left|I_{j}\right|}{\left|x-x_{j}\right|} .
$$

Because $\left\{I_{j}\right\}$ are Whitney intervals $\left|I_{j}\right| \lesssim\left|x-x_{j}\right|$ and $\ln ^{2}\left(\left|I_{j}\right| /\left|x-x_{j}\right|\right) \lesssim\left|x-x_{j}\right|^{\alpha} /\left|I_{j}\right|^{\alpha}$ for any $\alpha>0$, we deduce

$$
\int_{a_{j}-x}^{b_{j}-x} \int_{\left\{m \in L: x+m t \in I_{j}^{*}\right\}} \ln ^{2} \frac{1}{|m-1|} \mathrm{d} m \frac{\mathrm{d} t}{|t|} \lesssim \frac{\left|I_{j}\right|^{2-\alpha}}{\left|x-x_{j}\right|^{2-\alpha}} .
$$

As before

$$
\int_{a_{j}-x}^{b_{j}-x}\left|\frac{b(x+t)-b(x)}{t}\right|^{2} \frac{\mathrm{d} t}{|t|} \lesssim \frac{\left|I_{j}\right|^{3}}{\left|x-x_{j}\right|^{3}}
$$

We obtain

$$
\sum_{j} G_{j}(x) \lesssim \sum_{j} \frac{\left|I_{j}\right|^{2-\alpha}}{\left|x-x_{j}\right|^{2-\alpha}}
$$

where $0<\alpha<1$, which by (79) is finite a.e. $x \in F$.

It remains to estimate $H_{j}(x)$ for $x \in F \cap I$. Observe that $|t| \sim\left|x-x_{j}\right|$ if $t \in\left(a_{j}-x, b_{j}-x\right)$. Since $1<|m| \leq 2$ if the set

$$
J_{k}=\left\{m \in L: x+m t \in I_{k} \backslash I_{j}^{*}\right\}
$$

is nonempty we get that $\left|x-x_{k}\right| \simeq\left|x-x_{j}\right|$. Now we have

$$
\begin{aligned}
\int_{J_{k}} \frac{\mathrm{d} m}{(m-1)^{2}} & \lesssim\left|\left(\frac{a_{k}-x}{t}-1\right)^{-1}-\left(\frac{b_{k}-x}{t}-1\right)^{-1}\right| \\
& =\frac{|t|\left|b_{k}-a_{k}\right|}{\left|a_{k}-x-t\right|\left|b_{k}-x-t\right|} \approx \frac{|t|\left|I_{k}\right|}{\left(x_{k}-x_{j}\right)^{2}} .
\end{aligned}
$$


Since $b_{\mid F} \equiv 0$, condition (77) gives $|b(x+m t)| \lesssim\left|I_{k}\right|$ and $|b(x+t)| \lesssim\left|I_{j}\right|$ for any $t \in\left(a_{j}-x, b_{j}-x\right)$ and $m \in J_{k}$. Now, Fubini's theorem gives

$$
H_{j}(x) \lesssim \sum_{k \neq j:\left|x-x_{k}\right| \approx\left|x-x_{j}\right|}\left(\left|I_{k}\right|^{2}+\left|I_{j}\right|^{2}\right) \frac{\left|I_{k}\right|}{\left(x_{k}-x_{j}\right)^{2}} \int_{a_{j}-x}^{b_{j}-x} \frac{\mathrm{d} t}{|t|^{2}} .
$$

Denote by $A(j)$ the set of indices $k \neq j$ such that $\left|x-x_{k}\right| \approx\left|x-x_{j}\right|$. Since $\left\{I_{j}\right\}$ are Whitney intervals

$$
H_{j}(x) \lesssim \sum_{k \in A(j)} \frac{\left(\left|I_{k}\right|^{2}+\left|I_{j}\right|^{2}\right)\left|I_{k}\right|\left|I_{j}\right|}{\left(x_{k}-x_{j}\right)^{2}\left(x-x_{j}\right)^{2}}
$$

and since

$$
\sum_{k \in A(j)} \frac{\left|I_{k}\right|}{\left(x_{k}-x_{j}\right)^{2}} \lesssim \frac{1}{\left|I_{j}\right|}
$$

we get

$$
\sum_{k \in A(j)} \frac{\left|I_{j}\right|^{3}\left|I_{k}\right|}{\left(x_{k}-x_{j}\right)^{2}\left(x-x_{j}\right)^{2}} \lesssim \frac{\left|I_{j}\right|^{2}}{\left(x-x_{j}\right)^{2}}
$$

We have

$$
\sum_{j} \sum_{k \in A(j)} \frac{\left|I_{k}\right|^{3}\left|I_{j}\right|}{\left(x_{k}-x_{j}\right)^{2}\left(x-x_{j}\right)^{2}} \cong \sum_{k} \sum_{j \in A(k)} \frac{\left|I_{k}\right|^{3}\left|I_{j}\right|}{\left(x_{k}-x_{j}\right)^{2}\left(x-x_{k}\right)^{2}}
$$

and

$$
\sum_{j \in A(k)} \frac{\left|I_{j}\right|}{\left(x_{k}-x_{j}\right)^{2}} \lesssim \frac{1}{\left|I_{k}\right|}
$$

Therefore

$$
\sum_{j} \sum_{k \in A(j)} \frac{\left|I_{k}\right|^{3}\left|I_{j}\right|}{\left(x_{k}-x_{j}\right)^{2}\left(x-x_{j}\right)^{2}} \lesssim \sum_{k} \frac{\left|I_{k}\right|^{2}}{\left(x-x_{k}\right)^{2}}
$$

and so

$$
\sum_{j} H_{j}(x) \lesssim \sum_{j} \frac{\left|I_{j}\right|^{2}}{\left(x-x_{j}\right)^{2}}
$$

which by (79) is finite a.e. $x \in F$. This finishes the proof.

Acknowledgements J. Cufí, A. Nicolau and J. Verdera were partially supported by the Grants 2014SGR75 and 2014SGR289 of Generalitat de Catalunya, MTM2014-51824, MTM2013-44699, MTM2016-75390 and MTM2017-85666 of Ministerio de Educación, Cultura y Deporte. A. Seeger was partially supported by NSF Grant DMS 1500162, and as a Simons Visiting Researcher at CRM. He would like to thank the organizers of the 2016 program in Constructive Approximation and Harmonic Analysis for the invitation and for providing a pleasant and fruitful research atmosphere. 


\section{References}

1. Benedek, A., Calderón, A.-P., Panzone, R.: Convolution operators on Banach space valued functions. Proc. Natl. Acad. Sci. USA 48, 356-365 (1962)

2. Brezis, H.: How to recognize constant functions. Connections with Sobolev spaces. Uspekhi Mat. Nauk, 57(4) (346), 59-74 (2002). Russian Math. Surveys 57(4), 693-708 (2002)

3. Calderón, A.P.: Commutators of singular integrals. Proc. Natl. Acad. Sci. USA 53, 1092-1099 (1965)

4. Chang, S.Y.A., Fefferman, R.: A continuous version of duality of $H^{1}$ and $B M O$ on the bidisc. Ann. Math. 112, 179-201 (1980)

5. Christ, M.: Weak type $(1,1)$ bounds for rough operators. Ann. Math. 128, 19-42 (1988)

6. Donaire, J.J., Llorente, J.G., Nicolau, A.: Differentiability of functions in the Zygmund class. Proc. Lond. Math. Soc. (3) 108(1), 133-158 (2014)

7. Duoandikoetxea, J., Rubio de Francia, J.: Maximal and singular integral operators via Fourier transform estimates. Invent. Math. 84(3), 541-561 (1986)

8. Fefferman, C., Rivière, N.M., Sagher, Y.: Interpolation between $H^{p}$ spaces: the real method. Trans. Am. Math. Soc. 191, 75-81 (1974)

9. Hörmander, L.: Estimates for translation invariant operators in $L^{p}$ spaces. Acta Math. 104, 93-140 (1960)

10. Malý, J.: A simple proof of the Stepanov theorem on differentiability almost everywhere. Expo. Math. 17(1), 59-61 (1999)

11. Melnikov, M.S., Verdera, J.: A geometric proof of the $L^{2}$ boundedness of the Cauchy integral on Lipschitz graphs. Int. Math. Res. Not. (7), 325-331 (1995)

12. Peetre, J.: On spaces of Triebel-Lizorkin type. Ark. Mat. 13, 123-130 (1975)

13. Rubio de Francia, J.L., Ruiz, F.J., Torrea, J.L.: Calderón-Zygmund theory for operator-valued kernels. Adv. Math. 62(1), 7-48 (1986)

14. Seeger, A.: Remarks on singular convolution operators. Studia Math. 97, 91-114 (1990)

15. Seeger, A., Wright, J.: Problems on averages and lacunary maximal functions. Józef Marcinkiewicz Centenary Volume 95, 235-250 (2011)

16. Stein, E.M.: The characterization of functions arising as potentials. Bull. Am. Math. Soc. 67, 102-104 (1961)

17. Stein E.M. (1970) Singular integrals and differentiability properties of functions. Princeton Mathematical Series, No. 30. Princeton University Press, Princeton

18. Stein, E.M.: The development of square functions in the work of A. Zygmund. Bull. Am. Math. Soc. 7(2), 359-376 (1982)

19. Stein, E.M., Zygmund, A.: On the differentiability of functions. Studia Math. 23, 247-283 (1964)

20. Strichartz, R.: Multipliers on fractional Sobolev spaces. Indiana Univ. Math. J. (formerly J. Math. Mech.) 16(9), 1031-1060 (1967)

21. Triebel, H.: Theory of Function Spaces. II. Monographs in Mathematics, vol. 84. Birkhäuser Verlag, Basel (1992)

22. Triebel, H.: Tempered homogeneous function spaces. European Mathematical Society (EMS), Zürich, EMS Series of Lectures in Mathematics (2015)

23. Verdera, J.: $L^{2}$-boundedness of the Cauchy integral and Menger curvature. Harmonic analysis and boundary value problems (Fayetteville, AR, 2000), pp. 139-158, Contemp. Math., 277, Amer. Math. Soc., Providence, RI (2001) 